A. Mirzayeva, N. Slavinskaya, U. Riedel, M. Frenklach, A. Packard, W. Li, J. Oreluk, A. Hegde, Investigation of Dataset Construction Parameters and their Impact on Reaction Model Optimization using PrIMe, Proc. 56th AIAA Aerospace Sciences Meeting, 08.-12.01.2018, Kissimmee, USA AIAA 2018-0143

The AIAA version of the paper is accessible at https://doi.org/10.2514/6.2018-0143

On the AIAA web page http://www.aiaa.org/content.cfm?pageid=2

the interested reader can find other material published by AIAA 


\title{
Investigation of Dataset Construction Parameters and their Impact on Reaction Model Optimization using PrIMe
}

\author{
A. Mirzayeva ${ }^{1}$, N.A. Slavinskaya ${ }^{2}$, U. Riedel ${ }^{3}$ \\ Institute of Combustion Technology, German Aerospace Center (DLR), Stuttgart, 70569, Germany

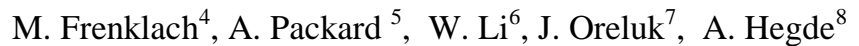 \\ Department of Mechanical Engineering, University of California, Berkeley, CA 94720, USA
}

\begin{abstract}
The current paper presents a continuation of the development of a modern methodology for the construction of uncertainty-quantified chemical reaction models on the base of the Bound-to-Bound Data Collaboration (B2BDC) module of the automated data-centric infrastructure PrIMe. Some problems, postulated in the recent studies, are in the focus of the present investigation. The question of targets amount (experimental data, Quantities of Interest (QoI)) selected for the analysis has been studied. To investigate this, the PrIMe dataset is augmented. The influence of dataset extension on the dataset consistency, feasible parameter set, and model optimization is studied and an algorithm for the selection of QoI in each experimental set is postulated. The approach of combined methods of scalar consistency measure, SCM, and vector consistency measure, VCM, for consistency analysis are adapted and successfully implemented. Predictions of the LS- $\mathcal{F}$ optimized mechanism are compared against a wide range of experimental data of laminar premixed flames and shock tube ignition delay times. Good agreement of model predictions with the experimental measurements is obtained.
\end{abstract}

$\begin{array}{ll}\phi & =\text { Nomenclature } \\ \phi & =\text { pressure behind reflected shock waves in shock-tube experiments } \\ P_{5} & =\text { quantity of interest } \\ Q o I & =\text { temperature behind reflected shock waves in shock-tube experiments } \\ T_{5} & =\text { initial temperature in laminar flame experiments } \\ T_{0} & =\text { uncertainty bounds }\end{array}$

\section{Introduction}

$\mathrm{O}$ $\mathrm{NE}$ of the most important properties of a reaction model in chemical engineering is to make predictions about the system when certain settings are changed. Appropriate handling and archiving of experimental data from different sources, and of the many uncertainties in the data embedded in the kinetic models, is a major challenge the chemical kinetics community has to tackle before becoming a predictive science. Methods for determining whether or not the model predictions are consistent with experimental data have been of great interest in combustion research over decades. Developing predictive models ${ }^{1}$ has become the goal in many of the modelling studies on reaction systems. Numerical optimization of complex reaction networks, of the kind that guided the development of GRIMech, ${ }^{1-3}$ e.g., has now been accepted as one of the underlying methods in this pursuit. ${ }^{4-6}$ In order to develop a

\footnotetext{
${ }^{1}$ PhD Student, Chemical Kinetics Department, Aziza.Mirzayeva@dlr.de

${ }^{2}$ Senior research fellow, Chemical Kinetics Department, Nadja.Slavinskaya@dlr.de, AIAA Senior Member

${ }^{3}$ Head of Chemical Kinetics Department, Uwe.Riedel@dlr.de, AIAA Senior Member

${ }^{4}$ Prof. of University of California, Berkeley, frenklach@berkeley.edu, AIAA Member

${ }^{5}$ Prof. of University of California, Berkeley, apackard@ berkeley.edu.

${ }^{6} \mathrm{PhD}$ Student of University of California, Berkeley, wenyuli@ berkeley.edu.

${ }^{7}$ PhD Student of University of California, Berkeley, jim.oreluk@ berkeley.edu.

${ }^{8} \mathrm{PhD}$ Student of University of California, Berkeley, arun.hegde@berkeley.edu.
} 
predictive reaction model for complex chemical systems the integration of large amounts of theoretical, computational, and experimental data collected by numerous researchers is required. The integration of such data entails assessment of the consistency of the data, validation of models, and quantification of uncertainties for model predictions and often requires calibrating unknown parameters based on experimental observations.

The interest for development of chemical models spurred the interest in the quality of experimental data, i.e. uncertainty quantification. There has been plenty of research on data collaboration over the years; ${ }^{1,2,7-9}$ this also led to the development of cybersystems offering kinetic database platforms, calculation and data analysis tools. Based on a long research on such cybersystems, we chose an automated data-centric infrastructure, the Process Informatics Model (PrIMe) ${ }^{7}$ and tested it on the updated version of hydrogen/syngas reaction model published in Slavinskaya et al. $^{10}$

\section{Theoretical background}

B2BDC is a computational framework developed for a treatment of collective information content (combined numerical and experimental data) collected from multiple sources. ${ }^{1,2,8,11-14}$ This approach allows the users to investigate the studied data consistency, identify the sources of inconsistency, evaluate (and re-evaluate) data uncertainty and optimize models under development. The main steps of the B2BDC methodology for the UQ reaction model analysis are dataset construction, feasible set computation, data consistency analysis and final model optimizations.

A dataset is a collection of model-data constraints where uncertain model parameters, with prior ranges $\left\{l_{i} \leq\right.$ $\left.x_{i} \leq u_{i}\right\}_{i=1}^{n}$, are constrained by both models $\left\{M_{e}(x)\right\}_{e=1}^{N}$ and experimental observations, or Quantities of Interest (QoIs), given on the uncertainty interval, $\left[L_{e}, U_{e}\right]$ for each $e$-th QoI. ${ }^{13-15}$ These models generally take the form of surrogates and map to observed experimental targets. Let the hyper-rectangle $\mathcal{H}$ define the prior uncertainty for the model parameters. For each individual QoI, we can find a feasible set of parameters on which the corresponding surrogate model matches the experimental bounds:

$$
\mathcal{F}_{e}:=\left\{x \in \mathcal{H}: L_{e} \leq M_{e}(x) \leq U_{e}\right\}
$$

The feasible set of the dataset is defined as follows:

$$
\mathcal{F}:=\cap_{e=1}^{N} \mathcal{F}_{e}=\left\{x \in \mathcal{H}: L_{e} \leq M_{e}(x) \leq U_{e}, \text { for } e=1, \ldots, N\right\}
$$

i.e., the collection of all parameters for which model predictions match experimental bounds. If the feasible set is nonempty, the dataset is said to be consistent. Conversely, if the feasible set is empty, i.e. inconsistent, then there is disagreement between models and data: no parameter vector can satisfy all model-data constraints. Inconsistency can often be attributed to misspecified experimental observations, incorrect model forms, or a combination of both.

Consistency analysis is a key unit of any dataset. If a dataset is defined to be inconsistent, no further analysis can be performed. Moreover, consistency of a dataset allows minimizing the error propagation. Therefore, prior to any model optimization and improvement, it is necessary to investigate the role of consistency analysis in the model optimization and influence of consistency assessment processes on the model output.

The B2BDC methodology offers two kinds of consistency analysis measures: SCM, namely scalar consistency measure, and VCM, vector consistency measure. In earlier work by R. Feeley and coworkers, ${ }^{11}$ a quantitative measure of dataset consistency was proposed as follows:

$$
\begin{gathered}
\text { SCM: }=\max _{x \in \mathcal{H}, \gamma} \gamma \\
\text { s.t. }(1-\gamma) \frac{L_{e}-U_{e}}{2} \leq M_{e}(x)-\frac{U_{e}+L_{e}}{2} \leq(1-\gamma) \frac{U_{e}-L_{e}}{2} \text { for } e=1, \ldots, N
\end{gathered}
$$

In recent work, ${ }^{16}$ this measure was termed the scalar consistency measure (SCM). Loosely speaking, the SCM provides the maximal allowable symmetric tightening of all model-data constraints. Hence, if the SCM is positive, the dataset is consistent. If the SCM is negative, then the dataset is inconsistent. In the case of inconsistent datasets, sensitivity calculations for the SCM provide a means of identifying possible problematic model-data constraints. Strategies for resolving inconsistent datasets using this technique are described in references. ${ }^{11,16}$ For massively inconsistent datasets (datasets with numerous contributors to inconsistency), these approaches can be overly 
aggressive and result in excessive identifications. To address this challenge, Hegde and coworkers proposed the vector consistency measure (VCM) for resolving massively inconsistent datasets through the finding the fewest number of relaxations of the QoI lower and upper bounds required to render the dataset consistency. ${ }^{16}$ Both strategies, SCM and VCM, are used in the present work for the data consistency analysis.

After a feasible set is constructed and dataset consistency is achieved, the reaction model optimization can be performed, e.g. through minimizing the sum of relative error between surrogate models and experimental data. ${ }^{17}$

Generally, three scenarios of optimization are available in PrIMe. The first method applied is LS- $\mathcal{H}$, a (weighted)

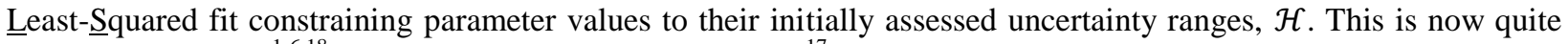
common approach. ${ }^{1-6,18}$ The other two refined methods, ${ }^{17} \mathrm{LS}-\mathcal{F}$ and $1 \mathrm{~N}-\mathcal{F}$, are constrained to the posterior uncertainty ranges of parameters, over the feasible set $\mathcal{F}$. Method LS-F $\mathcal{F}$ uses a weighted Least-Squared objective, while $1 \mathrm{~N}-\mathcal{F}$ is a $\underline{1}$-Norm minimization that aims at the smallest number of parameters to be changed.

In our recent research, ${ }^{10}$ the initial $\mathrm{H}_{2} / \mathrm{CO}$ reaction model, consisting of 73 reactions and 17 species, was subjected to a B2BDC analysis. A complete dataset including a total of 167 experimental targets (122 ignition delays, and 45 laminar flame speeds) and 55 active model parameters was constructed. Consistency analysis resulted in disagreement between model and data. Then, a final consistent dataset was composed by removing 45 experimental targets identified by VCM. Model optimization was performed with three methods, LS- $\mathcal{H}$, LS- $\mathcal{F}$ and $1 \mathrm{~N}-\mathcal{F}$. The LS- $\mathcal{F}$ model demonstrated the best agreement with experimental data resulting in zero number of the QoIs violation.

At the moment, it is unfortunately not guaranteed that a huge amount of data leads to a perfect well-predictive model, partly because the uncertainty prediction cannot be measured. In this work, we have augmented the dataset through a higher number of QoIs for syngas combustion by addition of more targets for already implemented ${ }^{10}$ and newly published experimental observation sets. The selection criterion is the minimum amount of units sufficient to reflect the experimental trend. Moreover, a new dataset now covers the hydrogen ignition delay times and laminar flame speed measurements. The influence of this expansion on the consistency analysis, feasible set, and model optimization is analyzed and compared to the previous research results.

Performed consistency analysis has motivated us to investigate the results obtained with SCM and VCM methods and their comparison. Furthermore, the action regarding the inconsistent constraints identified by VCM analysis, either their elimination or bound relaxation, has been studied.

\section{Workflow}

\section{A. Initial dataset}

The construction of a dataset is one of the main requirements of the B2BDC methodology. A dataset must consist of the experimental observations with respective uncertainty bounds, and a common reaction kinetic model with active parameters identified via sensitivity analysis. ${ }^{19}$

In the initial dataset, syngas and hydrogen ignition delay times and laminar flame speeds are considered. For ignition delay times, a set of shock-tube ignition delay time measurements is selected. It has a wide range of temperatures $(889-2136 \mathrm{~K})$, pressures $(0.6-33 \mathrm{bar})$, and equivalence ratios $(\varphi=0.5-4)$. To account for flamepropagation conditions, a set of flame velocity measurements is selected for preheat temperatures (298-700 K), pressures ( $0.5-40 \mathrm{bar})$, and equivalence ratios $(\varphi=0.5-5.6)$. These selected experimental observations cover the full range of operating conditions present in the literature sources. Experimental conditions of the selected measurements are summarized in Table 1. The uncertainty boundaries of the selected QoIs are assessed by evaluation with the empirical rule described in Slavinskaya et al. ${ }^{10}$ The final number of QoIs in the complete is 477 QoIs, including 319 ignition delays and 158 laminar flame speeds. The selected QoIs of ignition delay times and laminar flame speeds along with their evaluated uncertainties can be found in Appendixes 1-2.

\section{B. DLR_H $/$ SynG reaction kinetic model}

The chemical reaction kinetic model used in this research is a submodel of the DLR $\mathrm{C}_{0}-\mathrm{C}_{2}$ reaction model, ${ }^{10}$ which is the base chemistry of the DLR reaction data base for larger hydrocarbon oxidation.

\section{Ignition delay time determination}

Ignition delay time might be derived from different indicators: pressure change, temperature or species concentration (usually $\mathrm{OH}^{*}$ or $\mathrm{OH}$ ). PrIMe allows the user to store experimental data with information about the 
method used for ignition delay time determination. Nevertheless, the numerical simulation is not always able to identify uniquely the ignition delay time from $\mathrm{OH}^{*}$ gradient raise.

\begin{tabular}{|c|c|c|c|c|c|}
\hline \multicolumn{6}{|c|}{ Ignition delay time measurements } \\
\hline Authors & $\begin{array}{l}\text { Experimental } \\
\text { conditions }\end{array}$ & Authors & $\begin{array}{l}\text { Experimental } \\
\text { conditions }\end{array}$ & Authors & $\begin{array}{l}\text { Experimental } \\
\text { conditions }\end{array}$ \\
\hline${ }_{20}$ Kalitan et al. & $\begin{aligned} T_{5} & =900-1265 \mathrm{~K} ; \\
p_{5} & =0.6-17.9 \mathrm{bar} ; \\
\phi & =0.5\end{aligned}$ & Mathieu et al. $^{21}$ & $\begin{aligned} T_{5} & =998-2012 \mathrm{~K} ; \\
p_{5} & =1.6-32 \mathrm{~atm} ; \\
\phi & =0.5\end{aligned}$ & $\begin{array}{l}\text { Herzler } \quad \text { and } \\
\text { Naumann }^{22}\end{array}$ & $\begin{aligned} T_{5} & =918-1238 \mathrm{~K} ; \\
p_{5} & =1-16 \mathrm{~atm} ; \\
\phi & =0.5-1.0\end{aligned}$ \\
\hline $\begin{array}{l}\text { Petersen et } \\
\text { al. }^{23}\end{array}$ & $\begin{array}{l}T_{5}=943-1148 \mathrm{~K} ; \\
p_{5}=16.50-32.7 \mathrm{bar} ; \\
\phi=0.5\end{array}$ & Vasu et al. $^{24}$ & $\begin{aligned} T_{5} & =974-1160 \mathrm{~K} ; \\
p_{5} & =1.3-2.5 \mathrm{bar} ; \\
\phi & =0.5\end{aligned}$ & Naumann et al. ${ }^{25}$ & $\begin{array}{l}T_{5}=889-2136 \mathrm{~K} ; \\
p_{5}=1-16 \mathrm{~atm} ; \\
\phi=0.1-4.0\end{array}$ \\
\hline $\begin{array}{l}\text { Mertens et } \\
\text { al. }^{26}\end{array}$ & $\begin{aligned} T_{5} & =909-965 \mathrm{~K} \\
p_{5} & =1.2-1.4 \mathrm{bar} \\
\phi & =0.5-1.0\end{aligned}$ & Thi et al. $^{27}$ & $\begin{array}{l}T_{5}=902.1-1268.7 \\
\mathrm{~K} ; \\
p_{5}=2-20 \mathrm{~atm} ; \\
\phi=1.0-1.5\end{array}$ & Pan et al. ${ }^{28}$ & $\begin{array}{l}T_{5}=915.1-1456.7 \\
\mathrm{~K} ; \\
p_{5}=1.2 \mathrm{~atm} ; \\
\phi=1.0\end{array}$ \\
\hline Mittal et al. ${ }^{29}$ & $\begin{aligned} T_{5} & =1010.5-1044 \mathrm{~K} ; \\
p_{5} & =15-50 \text { bar; } \\
\phi & =1.0\end{aligned}$ & Petersen et al. ${ }^{30,31}$ & $\begin{array}{l}T_{5}=1189-1300 \mathrm{~K} ; \\
p_{5}=33 \mathrm{~atm} ; \\
\phi=1.0\end{array}$ & Zhang et al. ${ }^{32}$ & $\begin{array}{l}T_{5}=1023.52- \\
1267.23 \mathrm{~K} \\
p_{5}=5-20 \mathrm{~atm} ; \\
\phi=0.5\end{array}$ \\
\hline $\begin{array}{l}\text { Herzler and } \\
\text { Naumann }^{33}\end{array}$ & $\begin{array}{l}T_{5}=1019-1259 \mathrm{~K} ; \\
p_{5}=14.0-16.91 \mathrm{bar} ; \\
\phi=0.5\end{array}$ & Pang et al. ${ }^{34}$ & $\begin{aligned} T_{5} & =906-1118 \mathrm{~K} ; \\
p_{5} & =3.3-3.5 \mathrm{~atm} ; \\
\phi & =0.5\end{aligned}$ & Slack $^{35}$ & $\begin{array}{l}T_{5}=986.62- \\
1179.72 \mathrm{~K} \\
p_{5}=2 \mathrm{~atm} \\
\phi=1.0\end{array}$ \\
\hline Krejci et al. ${ }^{36}$ & $\begin{aligned} T_{5} & =980-2004 \mathrm{~K} ; \\
p_{5} & =1.6-32.0 \mathrm{bar} ; \\
\phi & =0.5\end{aligned}$ & & & & \\
\hline \multicolumn{6}{|c|}{ Laminar flame speed measurements } \\
\hline Sun et al. ${ }^{37}$ & $\begin{array}{l}T_{0}=298 \mathrm{~K} \\
p=0.5-40.0 \mathrm{~atm} \\
\phi=0.5-6.0\end{array}$ & Goswami et al. $^{38}$ & $\begin{array}{l}T_{0}=298 \mathrm{~K} ; \\
p=1-10 \mathrm{~atm} ; \\
\phi=0.5-0.6\end{array}$ & Wang et al. ${ }^{39}$ & $\begin{array}{l}T_{0}=298 \mathrm{~K} ; \\
p=1 \mathrm{~atm} ; \\
\phi=0.6-5.6\end{array}$ \\
\hline Hassan et al. ${ }^{40}$ & $\begin{array}{l}T_{0}=298 \mathrm{~K} \\
p=0.5-4.0 \mathrm{~atm} \\
\phi=0.6-5.0\end{array}$ & Zhang et al. ${ }^{41}$ & $\begin{array}{l}T_{0}=298 \mathrm{~K} ; \\
p=1-10 \mathrm{~atm} ; \\
\phi=0.5\end{array}$ & Weng et al. ${ }^{42}$ & $\begin{array}{l}T_{0}=295 \mathrm{~K} ; \\
p=1 \mathrm{~atm} ; \\
\phi=0.6-2.0\end{array}$ \\
\hline $\begin{array}{l}\text { Natarajan et } \\
\text { al. }\end{array}$ & $\begin{array}{l}T_{0}=300-700 \mathrm{~K} ; \\
p=1.0 \mathrm{~atm} ; \\
\phi=0.59-1.01\end{array}$ & Han et al. ${ }^{44}$ & $\begin{array}{l}T_{0}=298 \mathrm{~K} ; \\
p=5-10 \mathrm{~atm} ; \\
\phi=0.8\end{array}$ & Xie et al. ${ }^{45}$ & $\begin{array}{l}T_{0}=373 \mathrm{~K} ; \\
p=1-5 \mathrm{~atm} ; \\
\phi=0.6-3.0\end{array}$ \\
\hline $\begin{array}{l}\text { Natarajan et } \\
\text { al. }{ }^{46}\end{array}$ & $\begin{array}{l}T_{0}=600 \mathrm{~K} ; \\
p=15 \mathrm{~atm} ; \\
\phi=0.6-0.75\end{array}$ & $\begin{array}{l}\text { Lapalme and } \\
\text { Seers }\end{array}$ & $\begin{array}{l}T_{0}=295 \mathrm{~K} ; \\
p=1 \mathrm{~atm} ; \\
\phi=0.6-2.2\end{array}$ & Xie et al. ${ }^{48}$ & $\begin{array}{l}T_{0}=373 \mathrm{~K} ; \\
p=1 \mathrm{~atm} ; \\
\phi=0.6-3.0\end{array}$ \\
\hline Das et al. $^{49}$ & $\begin{array}{l}T_{0}=298-323 \mathrm{~K} ; \\
p=1.0 \mathrm{~atm} ; \\
\phi=0.6-1.2\end{array}$ & Li et al. ${ }^{50}$ & $\begin{array}{l}T_{0}=295 \mathrm{~K} ; \\
p=1 \mathrm{~atm} ; \\
\phi=0.6-1.0\end{array}$ & $\begin{array}{l}\text { Aung et al. } \\
\text { Taylor }^{52} \\
\text { Egolfopoulos and } \\
\text { Law }^{53} \\
\text { Law }^{54} \\
\text { Vagelopoulos et } \\
\text { al. }^{55} \\
\text { Hu et al. } \\
\text { Tang et al. }^{57} \\
\text { Verhelst et al. }^{58} \\
\text { Kwon and Faeth } \\
\text { Tse et al. } \\
\text { Ts }\end{array}$ & $\begin{array}{l}T_{0}=298 \mathrm{~K} ; \\
p=1 \mathrm{~atm} ; \\
\phi=0.5-4.5\end{array}$ \\
\hline $\begin{array}{l}\text { Goswami et } \\
\text { al. }{ }^{61}\end{array}$ & $\begin{array}{l}T_{0}=298 \mathrm{~K} ; \\
p=1-9 \mathrm{~atm} ; \\
\phi=0.6-1.0\end{array}$ & Sun et al. ${ }^{62}$ & $\begin{array}{l}T_{0}=400 \mathrm{~K} ; \\
p=1 \mathrm{~atm} ; \\
\phi=0.6-2.0\end{array}$ & Tse et al. ${ }^{60}$ & $\begin{array}{c}T_{0}=298 \mathrm{~K} ; \\
p=3-20 \mathrm{~atm} ; \\
\phi=0.6-3.5\end{array}$ \\
\hline
\end{tabular}

Table 1. Experimental measurements selected for initial dataset construction 
It becomes more critical for ignition modeling of syngas, which has various ignition regimes: strong, mixed, and transient. This might cause the phenomena of an 'unclear' ignition event, for instance saddle point in the initial phase (Fig. 1a); the multi-peak behavior of $\mathrm{OH}^{*}$ concentration profiles (Fig. 1b,c); peak s between the initial phase and steady state phase where the $\mathrm{OH}^{*}$ concentration is constant in spite of ongoing processes (Fig. 1c); or absence of the gradient peaks. These behaviors are demonstrated in Fig. 1a-f. An ideal case of gradient raise is shown in Fig. 2 .
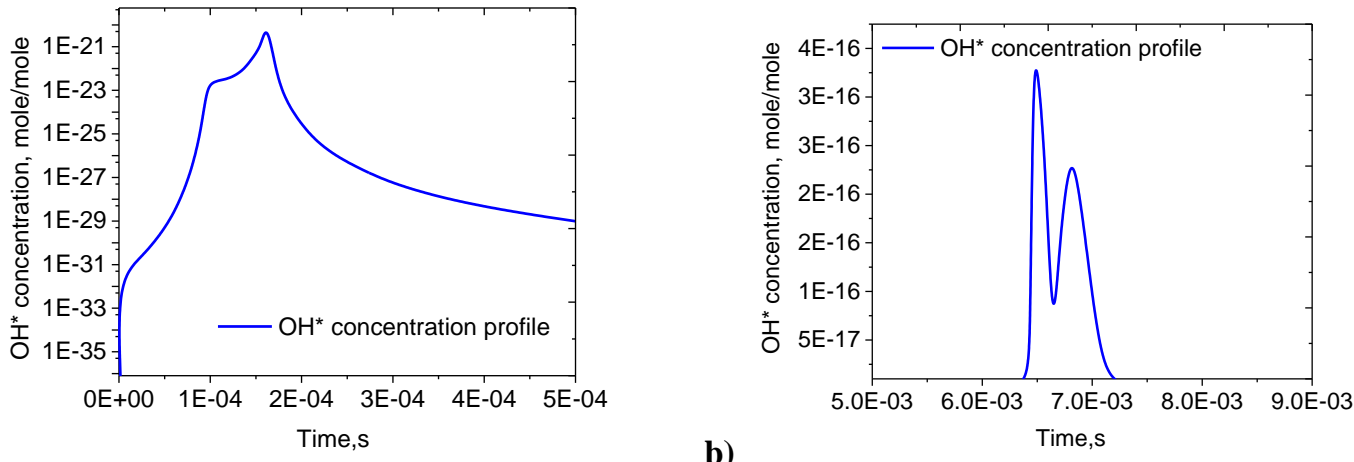

a)

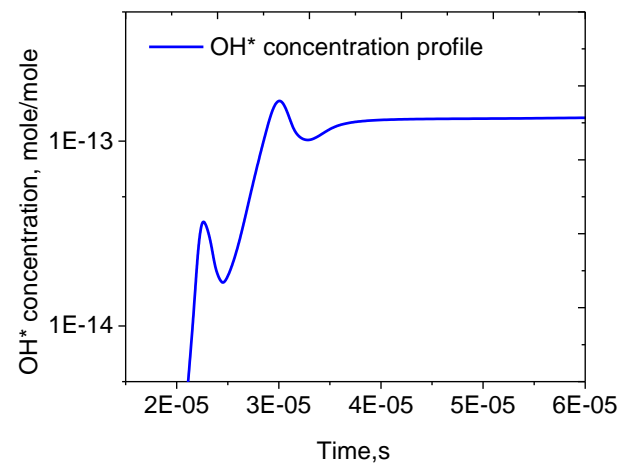

c)

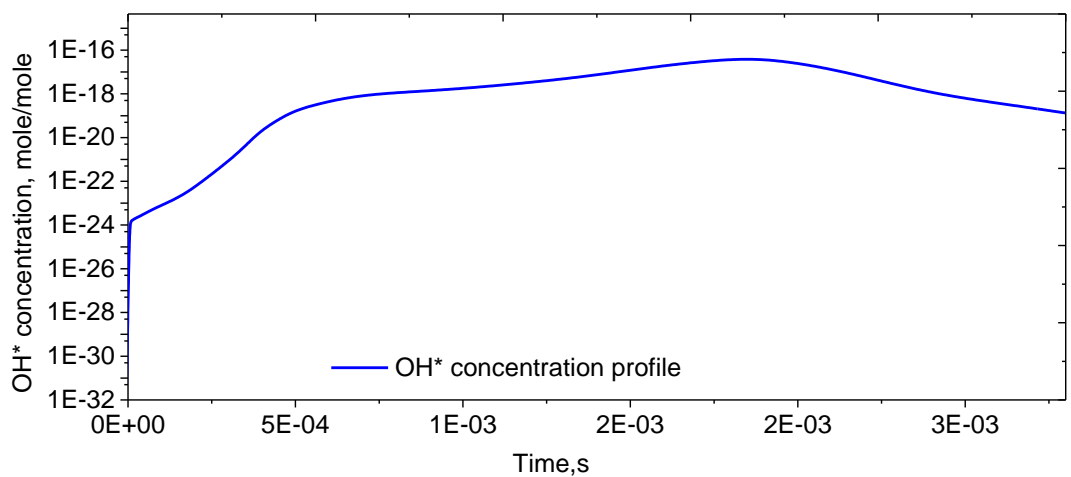

e)

b)

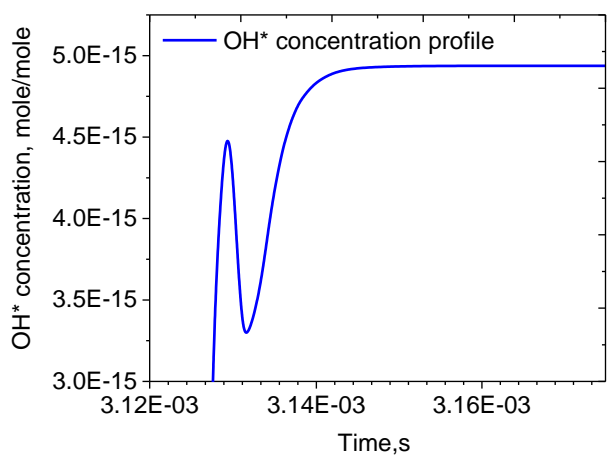

\section{Figure 1. Different behaviors of $\mathrm{OH}^{*}$ concentration profiles for ignition event determination}

The misinterpretation of numerical results (selection of the 'false' peak as ignition time points) leads to the confuse conclusion about agreement/disagreement of surrogate model with experimental value and, as result, to erroneous feasible set and difficulties with data consistency analysis. To address this problem, the following assumptions have been made:

- When there are two peaks in the $\mathrm{OH}^{*}$ profile as shown in Fig. 1a-c, the highest peak is used; 
- When the simulated steady state $\mathrm{OH}^{*}$ concentration is greater than the peak $\mathrm{OH}^{*}$ concentration as shown in Fig. 1d, the peak $\mathrm{OH}^{*}$ concentration is used;

- When there is no sharp gradient as shown in Fig. 1e, then the experimental point is assumed as self-inconsistent.

From the numerical point of view the $\mathrm{OH}^{*}$ concentration profile does not seem to be a good indicator for syngas ignition determination. We have compared the step rise according to both pressure and temperature time histories along with the $\mathrm{OH}^{*}$ concentration profile (Fig. 3a,b) for the QoI a00000238 with the initial model. It can be clearly seen that $\mathrm{OH}^{*}$ concentration profile gives two peaks whereas very similar ignition delay time of $0.0123 \mathrm{~s}$ is obtained from both pressure and temperature profiles. This allows us to assume that all three indicators for ignition time determination interchangeable and recommend using pressure and/or temperature profile as a unique indicator of ignition delay time for all QoIs.

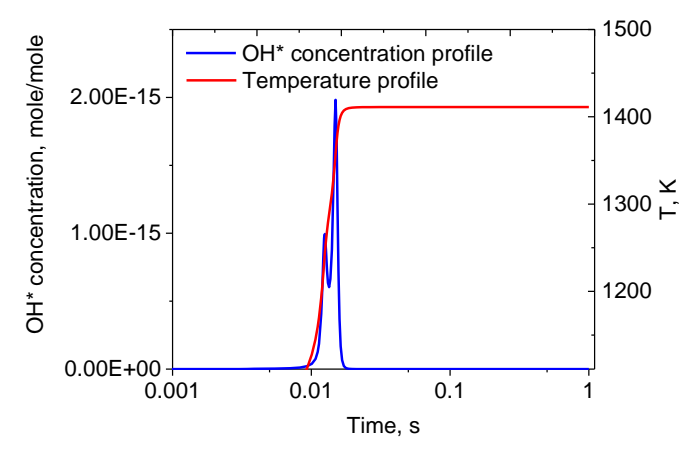

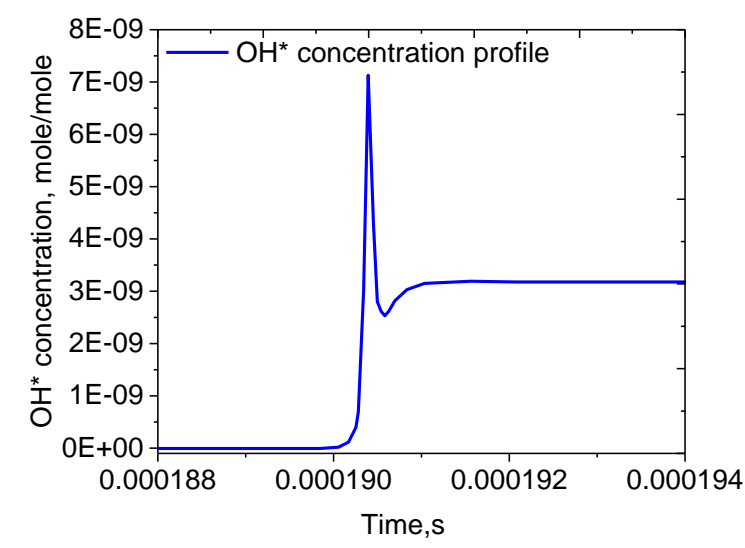

Figure 2. 'Ideal' case of $\mathrm{OH}^{*}$ concentration profile for ignition event determination

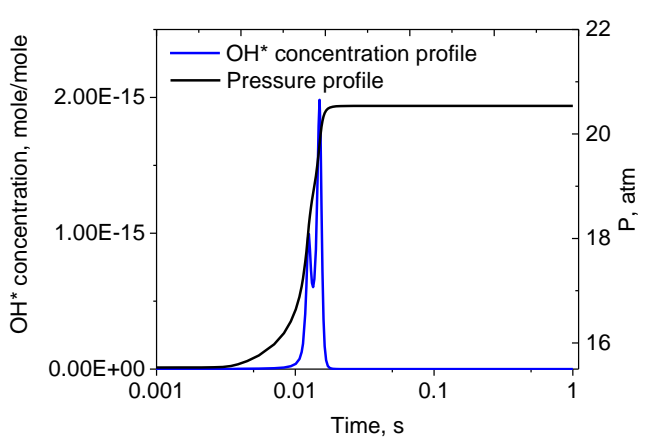

Figure 3. Comparison of different indicators for ignition time determination

\section{Preliminary filtering}

In order to provide more accurate fitting, a preliminary filtering is applied on the QoIs. According to the filtering results, eleven QoIs, a00000156, a00000157, a00000158, a00000309, a00000352, a00000355, a00000358, a00000359, a00000360, a00000701, and a00000504 (according to the case explained in Fig. 1e) are detected as selfinconsistent and removed before any further analysis. We assume that some of those QoIs stem from large deviation of modeled ignition time from the experimentally observed one, and some, perhaps, from the large ranges of parameter uncertainties.

\section{E. Construction of feasible region $F$}

The change in prior (original domain $\mathcal{H}$ ) and posterior (feasible set $\mathcal{F}$ ) parameter bounds for DLR_H $/$ /SynG dataset is demonstrated in Fig. 4. The variable index indicates the active model parameters. The full list of active parameters and their uncertainty bounds of rate coefficients is given in Slavinskaya et al. ${ }^{10}$ Uncertainty bounds are given as a relative value. Also shown, as black vertical lines, are prior parameter uncertainty bounds, whereas blue and red lines are the posterior inner and outer bounds, respectively.

\section{F. Consistency analysis}

After applying the preliminary filtration, a new dataset is generated after removing 11 self-inconsistent QoIs via preliminary filtering. This dataset, which includes 466 total QoIs, is originally inconsistent. After performing SCM analysis, the SCM interval $\gamma$ (Eq. 3) for the original dataset is found to be [-2.51, -2.31]. Since the SCM value is negative, the dataset is inconsistent. 


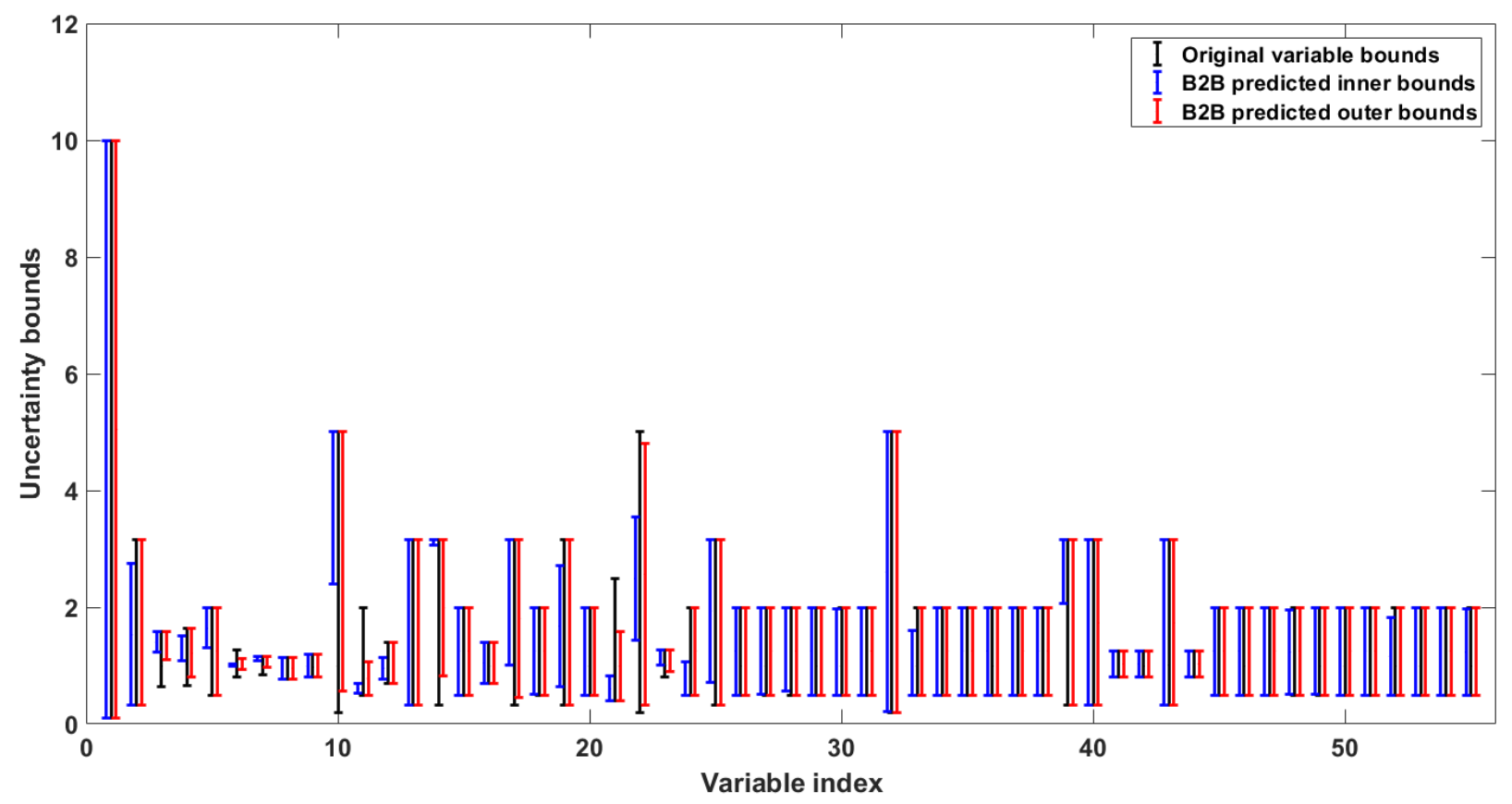

Figure 4. Prior and posterior parameter bounds

VCM analysis results in 87 targets to be relaxed or removed by VCM. These 87 QoIs consisting of 84 ignition delay QoIs and 3 flame speed QoIs are presented in Appendix 3. However, after the detailed analysis of all 87 QoIs, it has to be noted that this amount is overestimated, and mathematical algorithm still should be further investigated and improved. 87 QoIs are removed resulting in DLR_H $2 / S y n G$ dataset with SCM interval $\gamma$ equal to [0.047, 0.11]. The SCM is now positive, thus the final DLR_ $\mathrm{H}_{2} / \mathrm{SynG}$ dataset containing 379 QoIs is consistent.

\section{G. Parameter optimization and validation with experimental data}

Parameter optimization is designed to find an optimal point in the parameter space minimizing specified objectives. Generally, three types of optimization were applied:

1. LS- $\mathcal{F}$ - minimizing the sum of relative error between surrogate models and experimental data over the feasible set.

2. LS- $\mathcal{H}$ - minimizing the sum of relative error between surrogate models and experimental data over the original domain.

3. $1 \mathrm{~N}-\mathcal{F}-$ minimizing the 1 -norm of the parameter over the feasible set.

The data presented in Table 2 show a similar trend as in the previous research ${ }^{10}$ and highlight several features. According to the obtained results the lowest average deviation is shown by LS- $\mathcal{H}$ optimization. It was constrained to the prior uncertainty ranges of parameters, over the original domain $\mathcal{H}$. Nevertheless, LS- $\mathcal{H}$ results in violation of 28 QoIs uncertainty bounds. At the same time, the LS- $\mathcal{F}$ and $1 \mathrm{~N}-\mathcal{F}$ optimization methods, constrained to the posterior uncertainty ranges of parameters, over the feasible set $\mathcal{F}$, result in a lower average relative error and zero number of QoIs violation. The $1 \mathrm{~N}-\mathcal{F}$ scheme is using the least number of parameter changes, minimizing the 1norm of the parameter; therefore, it gives higher error as compared to the LS- $\mathcal{F}$. Thus, we can conclude that LS- $\mathcal{F}$ optimization method demonstrates the best predicting behavior among the three methods and will be used in the current study.

\begin{tabular}{|c|c|c|}
\hline $\begin{array}{c}\text { Optimization } \\
\text { method }\end{array}$ & Average error (\%) & $\begin{array}{c}\text { Number of QoIs } \\
\text { violations }\end{array}$ \\
\hline LS- $\mathcal{F}$ & 24.4 & 0 \\
\hline LS- $\mathcal{H}$ & 21.7 & 28 \\
\hline $1 \mathrm{~N}-\mathcal{F}$ & 40.6 & 0 \\
\hline
\end{tabular}

Table 2. Comparison of the optimization methods based on the surrogate models, pw 
A comparison between initial and final optimized model validated on experimental data is presented in Figures 5-6. Model predictions are also compared with those of Varga et al. ${ }^{6}$ since it was generated on the similar optimization method with much bigger amount of QoIs but without mathematical analysis of data consistency.
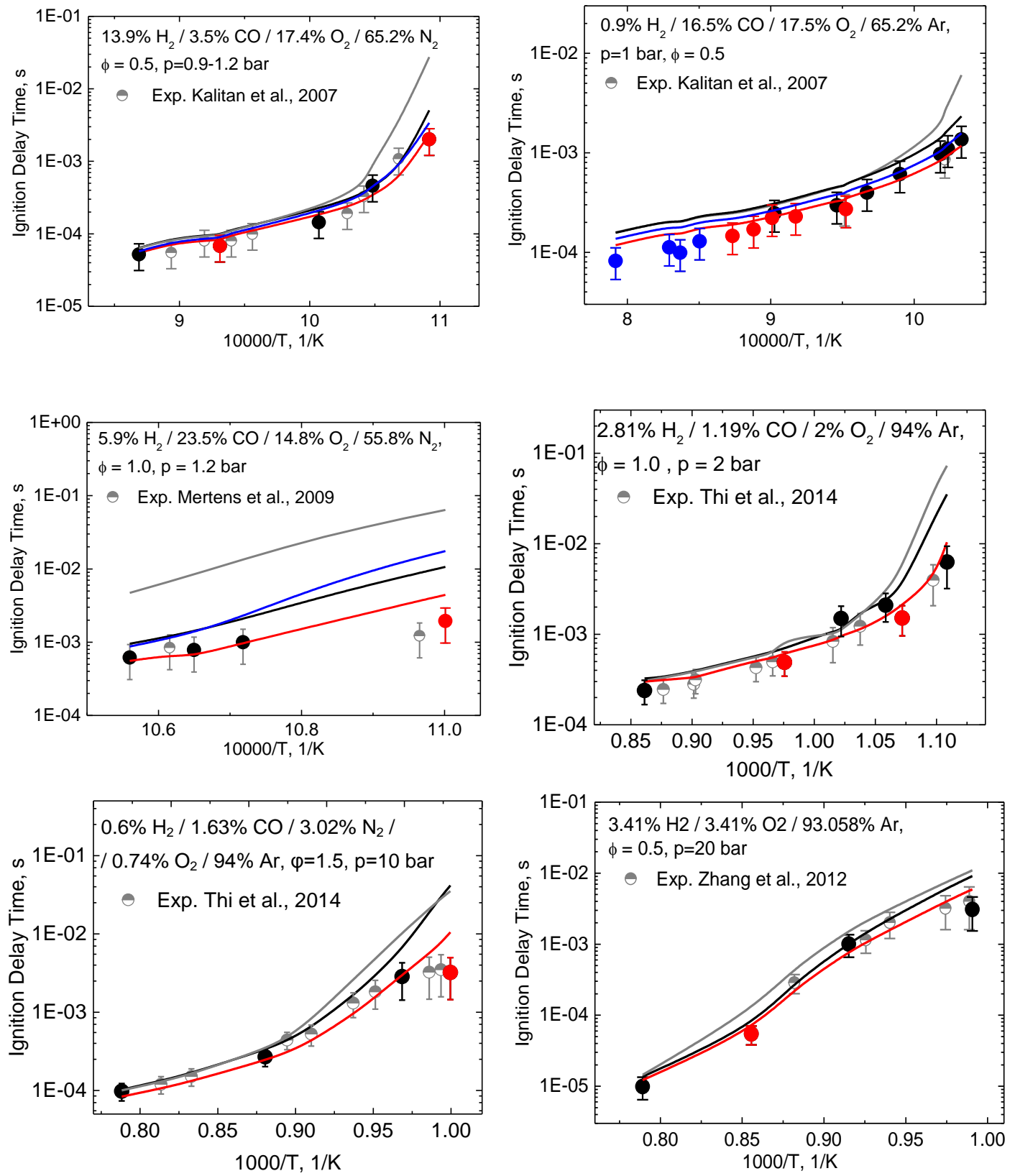

Figure 5. Syngas and hydrogen ignition delay time simulations. Symbols, experimental data; initial DLR model, black line; Varga et al. model, ${ }^{6}$ gray solid line; $L S-\mathcal{F}$ optimized model, $p w$, red solid line; $L S-\mathcal{F}$ optimized model, ${ }^{10}$ blue solid line; Black full circles are QoIs from DLR_H $H_{2} / \mathrm{Syn} G$ dataset; Red full circles are the QoIs deleted from the initial dataset according to the VCM analysis; Blue full circles are the self-inconsistent QoIs deleted from the initial dataset before any analysis.

Comparisons in Fig. 5 demonstrate that the predictions of the optimized model overall are in good agreement with the experimental measurements obtained in shock tubes. Fig. 6 shows the validation of initial and optimized models on the laminar flame speed data. The LS- $\mathcal{F}$ optimization method significantly improves model prediction accuracy against experimental results over low temperature regime of most significance in modeling ignition delay 
times. However, for some QoIs, there is no visible influence of the model optimization. Overall, the LS- $\mathcal{F}$ optimized model predicts the experimental data within the experimental uncertainties in case of consistent QoIs excluding those removed after VCM analysis (red circles with cross).

The results also demonstrate the influence of dataset augmentation. The comparison of LS- $\mathcal{F}$ models prediction applied on the dataset with total 122 QoIs $^{10}$ and 379 QoIs (pw) clearly indicates several points:

- From each experimental set, there should be sufficient amount of experimental points to reproduce the trend;

- Since the investigated reaction mechanism covers hydrogen and syngas combustion, construction of heterogeneous dataset (QoIs of both hydrogen and syngas) results in a higher confidence level of the model uncertainty quantification and, in consequence, better predictions of the optimized model.
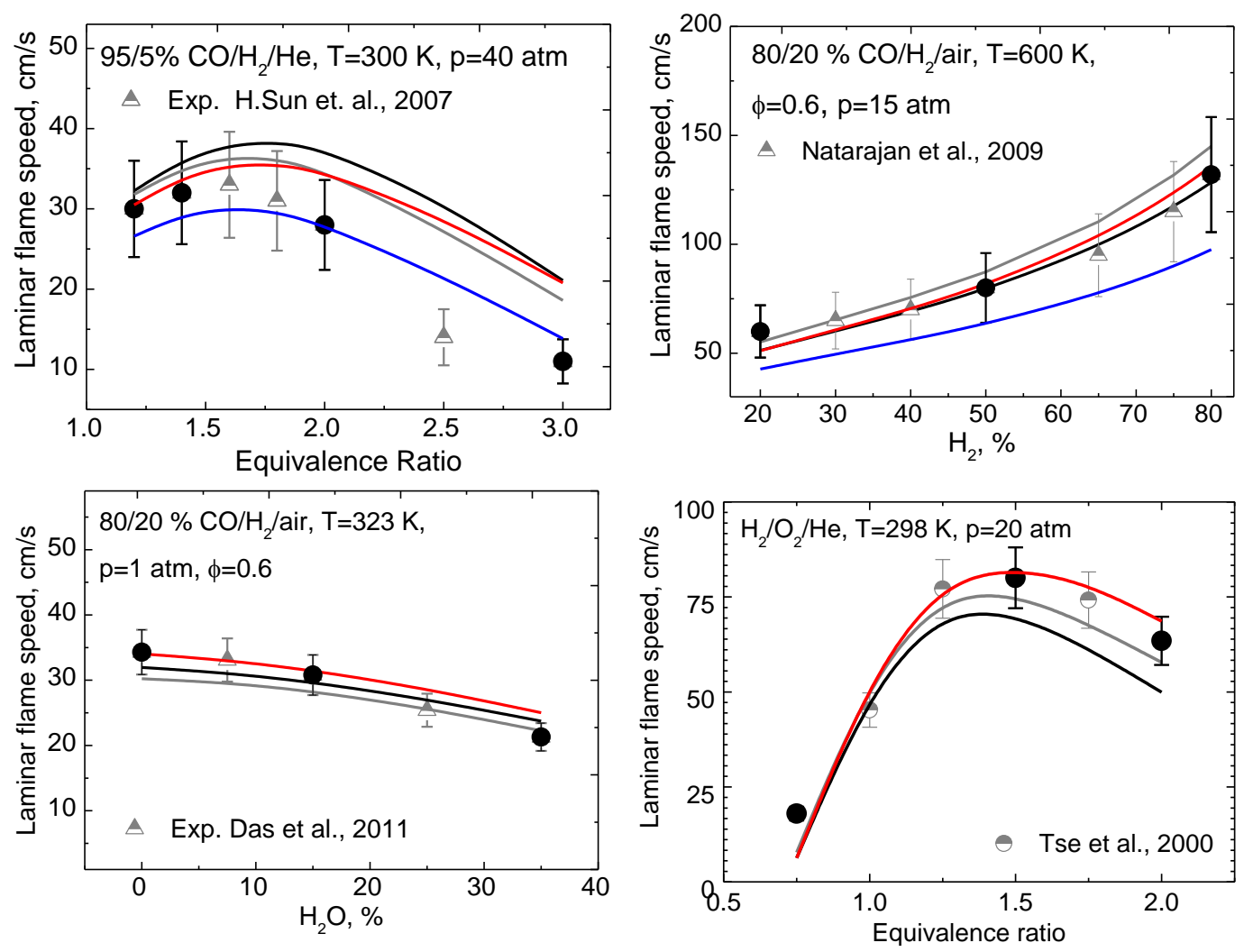

Figure 6. Syngas and hydrogen laminar flame speed simulations. Symbols, experimental data; initial DLR model, black line; Varga et al. model, ${ }^{6}$ gray solid line; $L S-\mathcal{F}$ optimized model, $p w$, red solid line; $L S-\mathcal{F}$ optimized model, ${ }^{10}$ blue solid line; Black full circles are QoIs from $D L R_{-} H_{2} /$ SynG dataset.

\section{Conclusion}

In this paper, B2BDC analysis of large amount of hydrogen and syngas combustion data was applied within the PrIMe cyber-infrastructure for investigation of dataset construction parameters and their impact on reaction model optimization.

The detailed syngas oxidation mechanism of Slavinskaya et al. ${ }^{10}$ has been updated using recently published rate constants. The approach of combined SCM and VCM methods for consistency analysis has been adapted from Hegde et al. ${ }^{16}$ and successfully implemented.

The problem of ignition delay determination based on $\mathrm{OH}^{*}$ concentration profile has been discussed and clarified. According to the research analysis, it has been recommended to use temperature and/or pressure time histories as a unique criterion of syngas ignition delay times for numerical simulations in PrIMe.

Dataset augmentation was performed and investigated. It has been found out that dataset augmentation has resulted in better prediction behavior of optimized model. 
Finally, predictions using the present LS- $\mathcal{F}$ optimized mechanism were compared against a wide range of experimental data of laminar premixed flames and shock tube ignition delay times. Improvement of optimized model prediction results can be clearly seen. Good agreement of model predictions with the experimental measurements allows us to further develop a complex uncertainty quantified $\mathrm{H}_{2}-\mathrm{CO}$ model for hydrogen and syngas combustion.

\section{Appendix}

Appendix 1. Ignition delay time QoIs

\begin{tabular}{|c|c|c|c|c|c|c|c|c|c|c|c|}
\hline \# & Mixture & $\mathrm{T}_{5}, \mathrm{~K}$ & $\begin{array}{l}\mathrm{P}_{5} \\
\text { bar }\end{array}$ & $\begin{array}{l}\text { Attribute } \\
\text { ID }\end{array}$ & $\begin{array}{c}\text { Estim } \\
\text { ated } \\
\text { error, } \\
\%\end{array}$ & \# & Mixture & $\mathrm{T}, \mathrm{K}$ & $\mathrm{p}$, bar & Attribute ID & $\begin{array}{c}\text { Estim } \\
\text { ated } \\
\text { error, } \\
\%\end{array}$ \\
\hline 1 & $40 / 60 \% \mathrm{CO} / \mathrm{H} 2 /$ air & 914.0 & 1.1 & a00000105 & 20 & 162 & $2.81 \% \mathrm{H} 2 / 1.19 \% \mathrm{CO} / 2 \% \mathrm{O} 2 / 94 \% \mathrm{Ar}$ & 1089.3 & 9.3 & $\mathrm{a} 00000558$ & 25 \\
\hline 2 & $40 / 60 \% \mathrm{CO} / \mathrm{H} 2 /$ air & 1036.0 & 1.1 & a00000106 & 20 & 163 & $2.81 \% \mathrm{H} 2 / 1.19 \% \mathrm{CO} / 2 \% \mathrm{O} 2 / 94 \% \mathrm{Ar}$ & 1061.5 & 9.2 & a00000559 & 35 \\
\hline 3 & 40/60\%CO/H2/air & 1241.0 & 1.0 & a00000107 & 20 & 164 & $2.81 \% \mathrm{H} 2 / 1.19 \% \mathrm{CO} / 2 \% \mathrm{O} 2 / 94 \% \mathrm{Ar}$ & 1020.5 & 10.0 & a00000560 & 50 \\
\hline 4 & 60/40\%CO/H2/air & 900.0 & 0.6 & $\mathrm{a} 00000110$ & 20 & 165 & $2.81 \% \mathrm{H} 2 / 1.19 \% \mathrm{CO} / 2 \% \mathrm{O} 2 / 94 \% \mathrm{Ar}$ & 937.7 & 9.5 & $\mathrm{a} 00000561$ & 60 \\
\hline 5 & 60/40\%CO/H2/air & 1026.0 & 1.1 & $\mathrm{a} 00000111$ & 20 & 166 & $2.81 \% \mathrm{H} 2 / 1.19 \% \mathrm{CO} / 2 \% \mathrm{O} 2 / 94 \% \mathrm{Ar}$ & 1182.9 & 19.5 & $\mathrm{a} 00000562$ & 30 \\
\hline 6 & $60 / 40 \% \mathrm{CO} / \mathrm{H} 2 / \mathrm{air}$ & 1162.0 & 1.0 & $\mathrm{a} 00000112$ & 20 & 167 & $2.81 \% \mathrm{H} 2 / 1.19 \% \mathrm{CO} / 2 \% \mathrm{O} 2 / 94 \% \mathrm{Ar}$ & 1097.1 & 20.3 & $\mathrm{a} 00000563$ & 35 \\
\hline 7 & $3.5 \% \mathrm{H} 2 / 14 \% \mathrm{CO} / 17.4 \% \mathrm{O} 2 / 65.1 \% \mathrm{Ar}$ & 936.0 & 1.2 & a00000113 & 20 & 168 & $2.81 \% \mathrm{H} 2 / 1.19 \% \mathrm{CO} / 2 \% \mathrm{O} 2 / 94 \% \mathrm{Ar}$ & 1039.1 & 20.0 & a00000564 & 50 \\
\hline 8 & $3.5 \% \mathrm{H} 2 / 14 \% \mathrm{CO} / 17.4 \% \mathrm{O} 2 / 65.1 \% \mathrm{Ar}$ & 1058.0 & 2.6 & a00000114 & 20 & 169 & $2.81 \% \mathrm{H} 2 / 1.19 \% \mathrm{CO} / 2 \% \mathrm{O} 2 / 94 \% \mathrm{Ar}$ & 1035.3 & 20.4 & a00000565 & 60 \\
\hline 9 & $3.5 \% \mathrm{H} 2 / 14 \% \mathrm{CO} / 17.4 \% \mathrm{O} 2 / 65.1 \% \mathrm{Ar}$ & 1190.0 & 16.8 & $\mathrm{a} 00000115$ & 20 & 170 & $2.81 \% \mathrm{H} 2 / 1.19 \% \mathrm{CO} / 2 \% \mathrm{O} 2 / 94 \% \mathrm{Ar}$ & 1024.4 & 17.2 & $\mathrm{a} 00000566$ & 75 \\
\hline 10 & $\begin{array}{l}1.7 \% \mathrm{H} 2 / 15.6 \% \mathrm{CO} / 17.5 \% \mathrm{O} 2 / 65.2 \% \\
\mathrm{Ar}\end{array}$ & 960.0 & 1.2 & a00000116 & 20 & 171 & $1.5 \% \mathrm{H} 2 / 3.0 \% \mathrm{CO} / 1.5 \% \mathrm{O} 2 / 94 \% \mathrm{Ar}$ & 1227.3 & 1.7 & $\mathrm{a} 00000567$ & 25 \\
\hline 11 & $\begin{array}{l}1.7 \% \mathrm{H} 2 / 15.6 \% \mathrm{CO} / 17.5 \% \mathrm{O} 2 / 65.2 \% \\
\mathrm{Ar}\end{array}$ & 1118.0 & 2.5 & a00000117 & 20 & 172 & $1.5 \% \mathrm{H} 2 / 3.0 \% \mathrm{CO} / 1.5 \% \mathrm{O} 2 / 94 \% \mathrm{Ar}$ & 1053.3 & 1.9 & a00000568 & 35 \\
\hline 12 & $\begin{array}{l}1.7 \% \mathrm{H} 2 / 15.6 \% \mathrm{CO} / 17.5 \% \mathrm{O} 2 / 65.2 \% \\
\mathrm{Ar}\end{array}$ & 1265.0 & 17.1 & a00000118 & 20 & 173 & $1.5 \% \mathrm{H} 2 / 3.0 \% \mathrm{CO} / 1.5 \% \mathrm{O} 2 / 94 \% \mathrm{Ar}$ & 945.1 & 1.8 & a00000569 & 45 \\
\hline 13 & $\begin{array}{l}0.9 \% \mathrm{H} 2 / 16.5 \% \mathrm{CO} / 17.5 \% \mathrm{O} 2 / 65.2 \% \\
\mathrm{Ar}\end{array}$ & 1121.0 & 14.0 & a00000121 & 20 & 174 & $1.5 \% \mathrm{H} 2 / 3.0 \% \mathrm{CO} / 1.5 \% \mathrm{O} 2 / 94 \% \mathrm{Ar}$ & 898.4 & 1.9 & a00000570 & 80 \\
\hline 14 & $40 / 60 \% \mathrm{CO} / \mathrm{H} 2 /$ air & 951.0 & 1.1 & a00000131 & 20 & 175 & $1.5 \% \mathrm{H} 2 / 3.0 \% \mathrm{CO} / 1.5 \% \mathrm{O} 2 / 94 \% \mathrm{Ar}$ & 1221.4 & 10.0 & a00000571 & 25 \\
\hline 15 & $40 / 60 \% \mathrm{CO} / \mathrm{H} 2 /$ air & 996.0 & 1.1 & a00000132 & 20 & 176 & $1.5 \% \mathrm{H} 2 / 3.0 \% \mathrm{CO} / 1.5 \% \mathrm{O} 2 / 94 \% \mathrm{Ar}$ & 1070.4 & 9.7 & a00000572 & 30 \\
\hline 16 & $40 / 60 \% \mathrm{CO} / \mathrm{H} 2 / \mathrm{air}$ & 1072.0 & 1.1 & a00000133 & 20 & 177 & $1.5 \% \mathrm{H} 2 / 3.0 \% \mathrm{CO} / 1.5 \% \mathrm{O} 2 / 94 \% \mathrm{Ar}$ & 1010.4 & 9.6 & a00000573 & 55 \\
\hline 17 & $40 / 60 \% \mathrm{CO} / \mathrm{H} 2 / \mathrm{air}$ & 1125.0 & 1.1 & $\mathrm{a} 00000134$ & 20 & 178 & $1.5 \% \mathrm{H} 2 / 3.0 \% \mathrm{CO} / 1.5 \% \mathrm{O} 2 / 94 \% \mathrm{Ar}$ & 1247.3 & 21.1 & $\mathrm{a} 00000574$ & 35 \\
\hline 18 & $40 / 60 \% \mathrm{CO} / \mathrm{H} 2 /$ air & 1175.0 & 1.0 & $\mathrm{a} 00000135$ & 20 & 179 & $1.5 \% \mathrm{H} 2 / 3.0 \% \mathrm{CO} / 1.5 \% \mathrm{O} 2 / 94 \% \mathrm{Ar}$ & 1133.0 & 19.9 & a00000575 & 30 \\
\hline 19 & 40/60\%CO/H2/air & 1187.0 & 1.0 & a00000136 & 20 & 180 & $1.5 \% \mathrm{H} 2 / 3.0 \% \mathrm{CO} / 1.5 \% \mathrm{O} 2 / 94 \% \mathrm{Ar}$ & 1046.0 & 20.2 & a00000576 & 60 \\
\hline 20 & $\begin{array}{l}0.9 \% \mathrm{H} 2 / 16.5 \% \mathrm{CO} / 17.5 \% \mathrm{O} 2 / 65.2 \% \\
\mathrm{Ar}\end{array}$ & 977.0 & 1.2 & a00000146 & 20 & 181 & $\begin{array}{l}1.54 \% \mathrm{H} 2 / 1.57 \% \mathrm{CO} / 1.34 \% \mathrm{CO} 2 / 1 . \\
55 \% \mathrm{O} 2 / 94 \% \mathrm{Ar}\end{array}$ & 1200.0 & 1.8 & a00000577 & 25 \\
\hline 21 & $\begin{array}{l}0.9 \% \mathrm{H} 2 / 16.5 \% \mathrm{CO} / 17.5 \% \mathrm{O} 2 / 65.2 \% \\
\mathrm{Ar}\end{array}$ & 982.0 & 1.1 & $\mathrm{a} 00000147$ & 20 & 182 & $\begin{array}{l}1.54 \% \mathrm{H} 2 / 1.57 \% \mathrm{CO} / 1.34 \% \mathrm{CO} 2 / 1 . \\
55 \% \mathrm{O} 2 / 94 \% \mathrm{Ar}\end{array}$ & 1064.1 & 1.8 & $\mathrm{a} 00000578$ & 30 \\
\hline 22 & $\begin{array}{l}0.9 \% \mathrm{H} 2 / 16.5 \% \mathrm{CO} / 17.5 \% \mathrm{O} 2 / 65.2 \% \\
\mathrm{Ar}\end{array}$ & 1010.0 & 1.2 & a00000148 & 20 & 183 & $\begin{array}{l}1.54 \% \mathrm{H} 2 / 1.57 \% \mathrm{CO} / 1.34 \% \mathrm{CO} 2 / 1 . \\
55 \% \mathrm{O} 2 / 94 \% \mathrm{Ar}\end{array}$ & 958.5 & 1.9 & $\mathrm{a} 00000579$ & 45 \\
\hline 23 & $\begin{array}{l}0.9 \% \mathrm{H} 2 / 16.5 \% \mathrm{CO} / 17.5 \% \mathrm{O} 2 / 65.2 \% \\
\mathrm{Ar}\end{array}$ & 1034.0 & 1.1 & a00000149 & 20 & 184 & $\begin{array}{l}1.54 \% \mathrm{H} 2 / 1.57 \% \mathrm{CO} / 1.34 \% \mathrm{CO} 2 / 1 . \\
55 \% \mathrm{O} 2 / 94 \% \mathrm{Ar}\end{array}$ & 915.6 & 1.9 & a00000580 & 60 \\
\hline 24 & $\begin{array}{l}0.9 \% \mathrm{H} 2 / 16.5 \% \mathrm{CO} / 17.5 \% \mathrm{O} 2 / 65.2 \% \\
\mathrm{Ar}\end{array}$ & 1050.0 & 1.1 & $\mathrm{a} 00000150$ & 20 & 185 & $\begin{array}{l}1.54 \% \mathrm{H} 2 / 1.57 \% \mathrm{CO} / 1.34 \% \mathrm{CO} 2 / 1 . \\
55 \% \mathrm{O} 2 / 94 \% \mathrm{Ar}\end{array}$ & 1257.6 & 9.8 & $\mathrm{a} 00000581$ & 25 \\
\hline 25 & $\begin{array}{l}0.9 \% \mathrm{H} 2 / 16.5 \% \mathrm{CO} / 17.5 \% \mathrm{O} 2 / 65.2 \% \\
\mathrm{Ar}\end{array}$ & 1108.0 & 1.1 & $\mathrm{a} 00000151$ & 20 & 186 & $\begin{array}{l}1.54 \% \mathrm{H} 2 / 1.57 \% \mathrm{CO} / 1.34 \% \mathrm{CO} 2 / 1 . \\
55 \% \mathrm{O} 2 / 94 \% \mathrm{Ar}\end{array}$ & 1101.9 & 9.6 & a00000582 & 30 \\
\hline 26 & $\begin{array}{l}0.9 \% \mathrm{H} 2 / 16.5 \% \mathrm{CO} / 17.5 \% \mathrm{O} 2 / 65.2 \% \\
\mathrm{Ar}\end{array}$ & 1090.0 & 1.1 & $\mathrm{a} 00000152$ & 20 & 187 & $\begin{array}{l}1.54 \% \mathrm{H} 2 / 1.57 \% \mathrm{CO} / 1.34 \% \mathrm{CO} 2 / 1 . \\
55 \% \mathrm{O} 2 / 94 \% \mathrm{Ar}\end{array}$ & 1055.7 & 9.8 & $\mathrm{a} 00000583$ & 35 \\
\hline 27 & $\begin{array}{l}0.9 \% \mathrm{H} 2 / 16.5 \% \mathrm{CO} / 17.5 \% \mathrm{O} 2 / 65.2 \% \\
\mathrm{Ar}\end{array}$ & 1110.0 & 1.1 & $\mathrm{a} 00000153$ & 20 & 188 & $\begin{array}{l}1.54 \% \mathrm{H} 2 / 1.57 \% \mathrm{CO} / 1.34 \% \mathrm{CO} 2 / 1 . \\
55 \% \mathrm{O} 2 / 94 \% \mathrm{Ar}\end{array}$ & 1036.2 & 8.2 & $\mathrm{a} 00000584$ & 40 \\
\hline 28 & $\begin{array}{l}0.9 \% \mathrm{H} 2 / 16.5 \% \mathrm{CO} / 17.5 \% \mathrm{O} 2 / 65.2 \% \\
\mathrm{Ar}\end{array}$ & 1126.0 & 1.1 & a00000154 & 20 & 189 & $\begin{array}{l}1.54 \% \mathrm{H} 2 / 1.57 \% \mathrm{CO} / 1.34 \% \mathrm{CO} 2 / 1 . \\
55 \% \mathrm{O} 2 / 94 \% \mathrm{Ar}\end{array}$ & 1026.8 & 10.2 & $\mathrm{a} 00000585$ & 55 \\
\hline 29 & $\begin{array}{l}0.9 \% \mathrm{H} 2 / 16.5 \% \mathrm{CO} / 17.5 \% \mathrm{O} 2 / 65.2 \% \\
\mathrm{Ar}\end{array}$ & 1145.0 & 1.1 & $\mathrm{a} 00000155$ & 20 & 190 & $\begin{array}{l}1.54 \% \mathrm{H} 2 / 1.57 \% \mathrm{CO} / 1.34 \% \mathrm{CO} 2 / 1 . \\
55 \% \mathrm{O} 2 / 94 \% \mathrm{Ar}\end{array}$ & 1015.6 & 10.1 & $\mathrm{a} 00000586$ & 50 \\
\hline 30 & $\begin{array}{l}0.9 \% \mathrm{H} 2 / 16.5 \% \mathrm{CO} / 17.5 \% \mathrm{O} 2 / 65.2 \% \\
\mathrm{Ar}\end{array}$ & 1176.0 & 1.0 & a00000156 & 20 & 191 & $\begin{array}{l}1.54 \% \mathrm{H} 2 / 1.57 \% \mathrm{CO} / 1.34 \% \mathrm{CO} 2 / 1 . \\
55 \% \mathrm{O} 2 / 94 \% \mathrm{Ar}\end{array}$ & 1238.6 & 20.2 & $\mathrm{a} 00000587$ & 35 \\
\hline 31 & $\begin{array}{l}0.9 \% \mathrm{H} 2 / 16.5 \% \mathrm{CO} / 17.5 \% \mathrm{O} 2 / 65.2 \% \\
\mathrm{Ar}\end{array}$ & 1195.0 & 1.1 & $\mathrm{a} 00000157$ & 20 & 192 & $\begin{array}{l}1.54 \% \mathrm{H} 2 / 1.57 \% \mathrm{CO} / 1.34 \% \mathrm{CO} 2 / 1 . \\
55 \% \mathrm{O} 2 / 94 \% \mathrm{Ar}\end{array}$ & 1151.3 & 20.0 & $\mathrm{a} 00000588$ & 30 \\
\hline 32 & $\begin{array}{l}0.9 \% \mathrm{H} 2 / 16.5 \% \mathrm{CO} / 17.5 \% \mathrm{O} 2 / 65.2 \% \\
\mathrm{Ar}\end{array}$ & 1206.0 & 1.0 & $\mathrm{a} 00000158$ & 20 & 193 & $\begin{array}{l}1.54 \% \mathrm{H} 2 / 1.57 \% \mathrm{CO} / 1.34 \% \mathrm{CO} 2 / 1 . \\
55 \% \mathrm{O} 2 / 94 \% \mathrm{Ar}\end{array}$ & 1103.7 & 20.2 & $\mathrm{a} 00000589$ & 40 \\
\hline 33 & $\begin{array}{l}0.9 \% \mathrm{H} 2 / 16.5 \% \mathrm{CO} / 17.5 \% \mathrm{O} 2 / 65.2 \% \\
\mathrm{Ar}\end{array}$ & 1005.0 & 2.3 & a00000159 & 20 & 194 & $\begin{array}{l}1.54 \% \mathrm{H} 2 / 1.57 \% \mathrm{CO} / 1.34 \% \mathrm{CO} 2 / 1 . \\
55 \% \mathrm{O} 2 / 94 \% \mathrm{Ar}\end{array}$ & 1051.0 & 19.3 & a00000590 & 55 \\
\hline 34 & 20/80\%CO/H2/air & 916.0 & 1.1 & a00000179 & 20 & 195 & $\begin{array}{l}0.6 \% \mathrm{H} 2 / 1.63 \% \mathrm{CO} / 3.02 \% \mathrm{~N} 2 / 0.74 \\
\% \mathrm{O} 2 / 94 \% \mathrm{Ar}\end{array}$ & 1175.8 & 1.9 & a00000591 & 25 \\
\hline 35 & 20/80\%CO/H2/air & 954.0 & 1.2 & $\mathrm{a} 00000181$ & 20 & 196 & $\begin{array}{l}0.6 \% \mathrm{H} 2 / 1.63 \% \mathrm{CO} / 3.02 \% \mathrm{~N} 2 / 0.74 \\
\% \mathrm{O} 2 / 94 \% \mathrm{Ar}\end{array}$ & 1039.4 & 1.9 & a00000592 & 35 \\
\hline 36 & 20/80\%CO/H2/air & 993.0 & 1.0 & a00000183 & 20 & 197 & $\begin{array}{l}0.6 \% \mathrm{H} 2 / 1.63 \% \mathrm{CO} / 3.02 \% \mathrm{~N} 2 / 0.74 \\
\% \mathrm{O} 2 / 94 \% \mathrm{Ar}\end{array}$ & 964.2 & 1.8 & a00000593 & 50 \\
\hline 37 & 20/80\%CO/H2/air & 1074.0 & 1.1 & $\mathrm{a} 00000186$ & 20 & 198 & $\begin{array}{l}0.6 \% \mathrm{H} 2 / 1.63 \% \mathrm{CO} / 3.02 \% \mathrm{~N} 2 / 0.74 \\
\% \mathrm{O} 2 / 94 \% \mathrm{Ar}\end{array}$ & 943.5 & 2.0 & a00000594 & 55 \\
\hline 38 & 20/80\%CO/H2/air & 1151.0 & 1.0 & a00000188 & 20 & 199 & $\begin{array}{l}0.6 \% \mathrm{H} 2 / 1.63 \% \mathrm{CO} / 3.02 \% \mathrm{~N} 2 / 0.74 \\
\% \mathrm{O} 2 / 94 \% \mathrm{Ar}\end{array}$ & 939.6 & 1.9 & a00000595 & 55 \\
\hline 39 & $3.5 \% \mathrm{H} 2 / 14 \% \mathrm{CO} / 17.4 \% \mathrm{O} 2 / 65.1 \% \mathrm{Ar}$ & 1015.0 & 1.1 & a00000189 & 20 & 200 & $\begin{array}{l}0.6 \% \mathrm{H} 2 / 1.63 \% \mathrm{CO} / 3.02 \% \mathrm{~N} 2 / 0.74 \\
\% \mathrm{O} 2 / 94 \% \mathrm{Ar}\end{array}$ & 940.2 & 2.0 & a00000596 & 70 \\
\hline 40 & $3.5 \% \mathrm{H} 2 / 14 \% \mathrm{CO} / 17.4 \% \mathrm{O} 2 / 65.1 \% \mathrm{Ar}$ & 1183.0 & 1.1 & a00000190 & 20 & 201 & $\begin{array}{l}0.6 \% \mathrm{H} 2 / 1.63 \% \mathrm{CO} / 3.02 \% \mathrm{~N} 2 / 0.74 \\
\% \mathrm{O} 2 / 94 \% \mathrm{Ar}\end{array}$ & 1268.7 & 10.2 & a00000597 & 25 \\
\hline 41 & $3.5 \% \mathrm{H} 2 / 14 \% \mathrm{CO} / 17.4 \% \mathrm{O} 2 / 65.1 \% \mathrm{Ar}$ & 929.0 & 2.6 & a00000191 & 20 & 202 & $\begin{array}{l}0.6 \% \mathrm{H} 2 / 1.63 \% \mathrm{CO} / 3.02 \% \mathrm{~N} 2 / 0.74 \\
\% \mathrm{O} 2 / 94 \% \mathrm{Ar}\end{array}$ & 1135.8 & 9.3 & a00000598 & 25 \\
\hline 42 & $3.5 \% \mathrm{H} 2 / 14 \% \mathrm{CO} / 17.4 \% \mathrm{O} 2 / 65.1 \% \mathrm{Ar}$ & 992.0 & 2.6 & a00000192 & 20 & 203 & $\begin{array}{l}0.6 \% \mathrm{H} 2 / 1.63 \% \mathrm{CO} / 3.02 \% \mathrm{~N} 2 / 0.74 \\
\% \mathrm{O} 2 / 94 \% \mathrm{Ar}\end{array}$ & 1032.5 & 10.3 & a00000599 & 50 \\
\hline
\end{tabular}

American Institute of Aeronautics and Astronautics 


\begin{tabular}{|c|c|c|c|c|c|c|c|c|c|c|c|}
\hline$\#$ & Mixture & $\mathrm{T}_{5}, \mathrm{~K}$ & $\begin{array}{l}\mathrm{P}_{5} \\
\text { bar }\end{array}$ & $\begin{array}{l}\text { Attribute } \\
\text { ID }\end{array}$ & $\begin{array}{c}\text { Estim } \\
\text { ated } \\
\text { error, } \\
\%\end{array}$ & \# & Mixture & $\mathrm{T}, \mathrm{K}$ & $\mathrm{p}, \mathrm{bar}$ & Attribute ID & $\begin{array}{c}\text { Estim } \\
\text { ated } \\
\text { error, } \\
\% \\
\end{array}$ \\
\hline 43 & $3.5 \% \mathrm{H} 2 / 14 \% \mathrm{CO} / 17.4 \% \mathrm{O} 2 / 65.1 \% \mathrm{Ar}$ & 1063.0 & 3.1 & a00000193 & 20 & 204 & $\begin{array}{l}0.6 \% \mathrm{H} 2 / 1.63 \% \mathrm{CO} / 3.02 \% \mathrm{~N} 2 / 0.74 \\
\% \mathrm{O} 2 / 94 \% \mathrm{Ar}\end{array}$ & 1000.4 & 10.1 & a00000600 & 55 \\
\hline 44 & $3.5 \% \mathrm{H} 2 / 14 \% \mathrm{CO} / 17.4 \% \mathrm{O} 2 / 65.1 \% \mathrm{Ar}$ & 1114.0 & 14.9 & a00000194 & 20 & 205 & $\begin{array}{l}0.6 \% \mathrm{H} 2 / 1.63 \% \mathrm{CO} / 3.02 \% \mathrm{~N} 2 / 0.74 \\
\% \mathrm{O} 2 / 94 \% \mathrm{Ar}\end{array}$ & 1226.9 & 19.7 & a00000601 & 25 \\
\hline 45 & $\begin{array}{l}1.7 \% \mathrm{H} 2 / 15.6 \% \mathrm{CO} / 17.5 \% \mathrm{O} 2 / 65.2 \% \\
\mathrm{Ar}\end{array}$ & 1052.0 & 1.1 & a00000195 & 20 & 206 & $\begin{array}{l}0.6 \% \mathrm{H} 2 / 1.63 \% \mathrm{CO} / 3.02 \% \mathrm{~N} 2 / 0.74 \\
\% \mathrm{O} 2 / 94 \% \mathrm{Ar}\end{array}$ & 1174.7 & 20.2 & a00000602 & 25 \\
\hline 46 & $\begin{array}{l}1.7 \% \mathrm{H} 2 / 15.6 \% \mathrm{CO} / 17.5 \% \mathrm{O} 2 / 65.2 \% \\
\mathrm{Ar}\end{array}$ & 1197.0 & 1.1 & a00000196 & 20 & 207 & $\begin{array}{l}0.6 \% \mathrm{H} 2 / 1.63 \% \mathrm{CO} / 3.02 \% \mathrm{~N} 2 / 0.74 \\
\% \mathrm{O} 2 / 94 \% \mathrm{Ar}\end{array}$ & 1133.9 & 20.0 & a00000603 & 30 \\
\hline 47 & $\begin{array}{l}1.7 \% \mathrm{H} 2 / 15.6 \% \mathrm{CO} / 17.5 \% \mathrm{O} 2 / 65.2 \% \\
\mathrm{Ar}\end{array}$ & 981.0 & 2.7 & a00000197 & 20 & 208 & $\begin{array}{l}0.6 \% \mathrm{H} 2 / 1.63 \% \mathrm{CO} / 3.02 \% \mathrm{~N} 2 / 0.74 \\
\% \mathrm{O} 2 / 94 \% \mathrm{Ar}\end{array}$ & 1104.3 & 20.5 & a00000604 & 40 \\
\hline 48 & $\begin{array}{l}1.7 \% \mathrm{H} 2 / 15.6 \% \mathrm{CO} / 17.5 \% \mathrm{O} 2 / 65.2 \% \\
\mathrm{Ar}\end{array}$ & 1048.0 & 2.5 & a00000198 & 20 & 209 & $\begin{array}{l}0.6 \% \mathrm{H} 2 / 1.63 \% \mathrm{CO} / 3.02 \% \mathrm{~N} 2 / 0.74 \\
\% \mathrm{O} 2 / 94 \% \mathrm{Ar}\end{array}$ & 1100.1 & 19.7 & a00000605 & 30 \\
\hline 49 & $\begin{array}{l}1.7 \% \mathrm{H} 2 / 15.6 \% \mathrm{CO} / 17.5 \% \mathrm{O} 2 / 65.2 \% \\
\mathrm{Ar}\end{array}$ & 1063.0 & 14.3 & a00000199 & 20 & 210 & $\begin{array}{l}0.6 \% \mathrm{H} 2 / 1.63 \% \mathrm{CO} / 3.02 \% \mathrm{~N} 2 / 0.74 \\
\% \mathrm{O} 2 / 94 \% \mathrm{Ar}\end{array}$ & 1098.1 & 20.9 & a00000606 & 45 \\
\hline 50 & $\begin{array}{l}1.7 \% \mathrm{H} 2 / 15.6 \% \mathrm{CO} / 17.5 \% \mathrm{O} 2 / 65.2 \% \\
\mathrm{Ar}\end{array}$ & 1126.0 & 11.9 & a00000200 & 20 & 211 & $\begin{array}{l}0.6 \% \mathrm{H} 2 / 1.63 \% \mathrm{CO} / 3.02 \% \mathrm{~N} 2 / 0.74 \\
\% \mathrm{O} 2 / 94 \% \mathrm{Ar}\end{array}$ & 1073.2 & 20.3 & a00000607 & 50 \\
\hline 51 & $\begin{array}{l}0.9 \% \mathrm{H} 2 / 16.5 \% \mathrm{CO} / 17.5 \% \mathrm{O} 2 / 65.2 \% \\
\mathrm{Ar}\end{array}$ & 1114.0 & 2.1 & a00000203 & 20 & 212 & $2 \% \mathrm{H} 2 / 1 \% \mathrm{O} 2 / 97 \% \mathrm{Ar}$ & 1300.0 & 33.0 & a00000608 & 45 \\
\hline 52 & $\begin{array}{l}0.9 \% \mathrm{H} 2 / 16.5 \% \mathrm{CO} / 17.5 \% \mathrm{O} 2 / 65.2 \% \\
\mathrm{Ar}\end{array}$ & 1074.0 & 19.2 & $\mathrm{a} 00000205$ & 20 & 213 & $2 \% \mathrm{H} 2 / 1 \% \mathrm{O} 2 / 97 \% \mathrm{Ar}$ & 1264.0 & 33.0 & a00000609 & 45 \\
\hline 53 & $\begin{array}{l}0.9 \% \mathrm{H} 2 / 16.5 \% \mathrm{CO} / 17.5 \% \mathrm{O} 2 / 65.2 \% \\
\mathrm{Ar}\end{array}$ & 1114.0 & 14.6 & a00000206 & 20 & 214 & $2 \% \mathrm{H} 2 / 1 \% \mathrm{O} 2 / 97 \% \mathrm{Ar}$ & 1221.0 & 33.0 & a00000610 & 40 \\
\hline 54 & \begin{tabular}{|l|}
$7.33 \% \mathrm{H} 2 / 9.71 \% \mathrm{CO} / 1.98 \% \mathrm{CO} 2 / 17.0$ \\
$1 \% \mathrm{O} 2 / 63.97 \% \mathrm{~N} 2$
\end{tabular} & 1013.0 & 21.6 & a00000208 & 20 & 215 & $2 \% \mathrm{H} 2 / 1 \% \mathrm{O} 2 / 97 \% \mathrm{Ar}$ & 1189.0 & 33.0 & a00000611 & 40 \\
\hline 55 & $0.5 \% \mathrm{H} 2 / 14 \% \mathrm{CO} / 17.4 \% \mathrm{O} 2 / 65.1 \% \mathrm{Ar}$ & 1015.0 & 13.7 & a00000213 & 20 & 216 & $4 \% \mathrm{H} 2 / 2 \% \mathrm{O} 2 / 94 \% \mathrm{Ar}$ & 930.0 & 3.6 & a00000612 & 45 \\
\hline 56 & $80 / 20 \% \mathrm{CO} / \mathrm{H} 2 /$ air & 939.0 & 1.1 & a00000221 & 30 & 217 & $4 \% \mathrm{H} 2 / 2 \% \mathrm{O} 2 / 94 \% \mathrm{Ar}$ & 938.0 & 3.5 & a00000613 & 40 \\
\hline 57 & $\begin{array}{l}15.6 \% \mathrm{CO} / 1.7 \% \mathrm{H} 2 / 17.5 \% \mathrm{O} 2 / 65.2 \% \\
\mathrm{Ar}\end{array}$ & 932.0 & 1.4 & a00000223 & 30 & 218 & $4 \% \mathrm{H} 2 / 2 \% \mathrm{O} 2 / 94 \% \mathrm{Ar}$ & 964.0 & 3.6 & a00000614 & 35 \\
\hline 58 & $\begin{array}{l}15.6 \% \mathrm{CO} / 1.7 \% \mathrm{H} 2 / 17.5 \% \mathrm{O} 2 / 65.2 \% \\
\mathrm{Ar}\end{array}$ & 956.0 & 1.4 & a00000224 & 30 & 219 & $4 \% \mathrm{H} 2 / 2 \% \mathrm{O} 2 / 94 \% \mathrm{Ar}$ & 987.0 & 3.4 & a00000615 & 30 \\
\hline 59 & $\begin{array}{l}15.6 \% \mathrm{CO} / 1.7 \% \mathrm{H} 2 / 17.5 \% \mathrm{O} 2 / 65.2 \% \\
\mathrm{Ar}\end{array}$ & 965.0 & 1.5 & a00000225 & 30 & 220 & $4 \% \mathrm{H} 2 / 2 \% \mathrm{O} 2 / 94 \% \mathrm{Ar}$ & 1021.0 & 3.4 & a00000616 & 20 \\
\hline 60 & $\begin{array}{l}0.436 \% \mathrm{H} 2 / 8.228 \% \mathrm{CO} / 8.657 \% \mathrm{O} 2 / 82 . \\
679 \% \mathrm{Ar}\end{array}$ & 1046.0 & 16.8 & a00000226 & 20 & 221 & $4 \% \mathrm{H} 2 / 2 \% \mathrm{O} 2 / 94 \% \mathrm{Ar}$ & 1118.0 & 3.4 & a00000617 & 20 \\
\hline 61 & $\begin{array}{l}0.436 \% \mathrm{H} 2 / 8.228 \% \mathrm{CO} / 8.657 \% \mathrm{O} 2 / 82 . \\
679 \% \mathrm{Ar}\end{array}$ & 1072.0 & 15.8 & a00000227 & 20 & 222 & $15 \% \mathrm{H} 2 / 18 \% \mathrm{O} 2 / 67 \% \mathrm{Ar}$ & 908.0 & 3.4 & a00000618 & 40 \\
\hline 62 & $\begin{array}{l}0.436 \% \mathrm{H} 2 / 8.228 \% \mathrm{CO} / 8.657 \% \mathrm{O} 2 / 82 . \\
679 \% \mathrm{Ar}\end{array}$ & 1132.0 & 16.2 & $\mathrm{a} 00000228$ & 20 & 223 & $15 \% \mathrm{H} 2 / 18 \% \mathrm{O} 2 / 67 \% \mathrm{Ar}$ & 919.0 & 3.4 & a00000619 & 40 \\
\hline 63 & $\begin{array}{l}0.175 \% \mathrm{H} 2 / 3.301 \% \mathrm{CO} / 3.473 \% \mathrm{O} 2 / 93 \\
051 \% \mathrm{Ar}\end{array}$ & 1107.0 & 16.2 & a00000229 & 20 & 224 & $15 \% \mathrm{H} 2 / 18 \% \mathrm{O} 2 / 67 \% \mathrm{Ar}$ & 951.0 & 3.3 & a00000620 & 30 \\
\hline 64 & $\begin{array}{l}0.175 \% \mathrm{H} 2 / 3.301 \% \mathrm{CO} / 3.473 \% \mathrm{O} 2 / 93 \\
051 \% \mathrm{Ar}\end{array}$ & 1159.0 & 16.1 & $\mathrm{a} 00000230$ & 20 & 225 & $15 \% \mathrm{H} 2 / 18 \% \mathrm{O} 2 / 67 \% \mathrm{Ar}$ & 992.0 & 3.2 & a00000621 & 25 \\
\hline 65 & \begin{tabular}{|l|}
$0.175 \% \mathrm{H} 2 / 3.301 \% \mathrm{CO} / 3.473 \% \mathrm{O} 2 / 93$ \\
$051 \% \mathrm{Ar}$
\end{tabular} & 1206.0 & 16.4 & a00000231 & 20 & 226 & $15 \% \mathrm{H} 2 / 18 \% \mathrm{O} 2 / 67 \% \mathrm{Ar}$ & 1049.0 & 3.0 & a00000622 & 25 \\
\hline 66 & $\begin{array}{l}0.088 \% \mathrm{H} 2 / 1.654 \% \mathrm{CO} / 1.740 \% \mathrm{O} 2 / 96 \\
518 \mathrm{Ar}\end{array}$ & 1165.0 & 16.1 & $\mathrm{a} 00000232$ & 20 & 227 & $3.46 \% \mathrm{H} 2 / 3.49 \% \mathrm{O} 2 / 93.05 \% \mathrm{Ar}$ & 923.0 & 1.0 & a00000623 & 35 \\
\hline 67 & $\begin{array}{l}0.088 \% \mathrm{H} 2 / 1.654 \% \mathrm{CO} / 1.740 \% \mathrm{O} 2 / 96 . \\
518 \mathrm{Ar}\end{array}$ & 1207.0 & 16.4 & a00000233 & 20 & 228 & $3.46 \% \mathrm{H} 2 / 3.49 \% \mathrm{O} 2 / 93.05 \% \mathrm{Ar}$ & 934.0 & 1.1 & a00000624 & 35 \\
\hline 68 & $\begin{array}{l}0.088 \% \mathrm{H} 2 / 1.654 \% \mathrm{CO} / 1.740 \% \mathrm{O} 2 / 96 . \\
518 \mathrm{Ar}\end{array}$ & 1259.0 & 15.9 & a00000234 & 20 & 229 & $3.46 \% \mathrm{H} 2 / 3.49 \% \mathrm{O} 2 / 93.05 \% \mathrm{Ar}$ & 941.0 & 1.1 & a00000625 & 30 \\
\hline 69 & $\begin{array}{l}4.364 \% \mathrm{H} 2 / 4.364 \% \mathrm{CO} / 8.718 \% \mathrm{O} 2 / 82 . \\
554 \% \mathrm{Ar}\end{array}$ & 1019.0 & 14.1 & a00000235 & 20 & 230 & $3.46 \% \mathrm{H} 2 / 3.49 \% \mathrm{O} 2 / 93.05 \% \mathrm{Ar}$ & 1027.0 & 1.0 & a00000626 & 20 \\
\hline 70 & $\begin{array}{l}4.364 \% \mathrm{H} 2 / 4.364 \% \mathrm{CO} / 8.718 \% \mathrm{O} 2 / 82 . \\
554 \% \mathrm{Ar}\end{array}$ & 1051.0 & 15.3 & a00000236 & 20 & 231 & $5.87 \% \mathrm{H} 2 / 2.95 \% \mathrm{O} 2 / 91.18 \% \mathrm{Ar}$ & 918.0 & 1.0 & a00000627 & 35 \\
\hline 71 & $\begin{array}{l}4.364 \% \mathrm{H} 2 / 4.364 \% \mathrm{CO} / 8.718 \% \mathrm{O} 2 / 82 . \\
554 \% \mathrm{Ar}\end{array}$ & 1097.0 & 15.6 & a00000237 & 20 & 232 & $5.87 \% \mathrm{H} 2 / 2.95 \% \mathrm{O} 2 / 91.18 \% \mathrm{Ar}$ & 956.0 & 0.9 & a00000628 & 30 \\
\hline 72 & $\begin{array}{l}1.77 \% \mathrm{H} 2 / 1.80 \% \mathrm{CO} / 3.559 \% \mathrm{O} 2 / 92.87 \\
\% \mathrm{Ar}\end{array}$ & 1048.0 & 16.0 & a00000238 & 20 & 233 & $5.87 \% \mathrm{H} 2 / 2.95 \% \mathrm{O} 2 / 91.18 \% \mathrm{Ar}$ & 1061.0 & 1.0 & a00000629 & 20 \\
\hline 73 & $\begin{array}{l}1.77 \% \mathrm{H} 2 / 1.80 \% \mathrm{CO} / 3.559 \% \mathrm{O} 2 / 92.87 \\
\% \mathrm{Ar}\end{array}$ & 1086.0 & 15.5 & a00000239 & 20 & 234 & $5.87 \% \mathrm{H} 2 / 2.95 \% \mathrm{O} 2 / 91.18 \% \mathrm{Ar}$ & 1718.0 & 1.1 & a00000630 & 30 \\
\hline 74 & $\begin{array}{l}1.77 \% \mathrm{H} 2 / 1.80 \% \mathrm{CO} / 3.559 \% \mathrm{O} 2 / 92.87 \\
\% \mathrm{Ar}\end{array}$ & 1128.0 & 15.5 & a00000240 & 20 & 235 & $3.46 \% \mathrm{H} 2 / 3.49 \% \mathrm{O} 2 / 93.05 \% \mathrm{Ar}$ & 958.0 & 3.9 & a00000631 & 35 \\
\hline 75 & $\begin{array}{l}0.868 \% \mathrm{H} 2 / 0.868 \% \mathrm{CO} / 1.736 \% \mathrm{O} 2 / 96 \\
529 \% \mathrm{Ar}\end{array}$ & 1054.0 & 15.6 & a00000241 & 20 & 236 & $3.46 \% \mathrm{H} 2 / 3.49 \% \mathrm{O} 2 / 93.05 \% \mathrm{Ar}$ & 992.0 & 4.0 & a00000632 & 30 \\
\hline 76 & $\begin{array}{l}0.868 \% \mathrm{H} 2 / 0.868 \% \mathrm{CO} / 1.736 \% \mathrm{O} 2 / 96 \\
529 \% \mathrm{Ar}\end{array}$ & 1090.0 & 15.8 & a00000242 & 20 & 237 & $3.46 \% \mathrm{H} 2 / 3.49 \% \mathrm{O} 2 / 93.05 \% \mathrm{Ar}$ & 1035.0 & 3.8 & a00000633 & 20 \\
\hline 77 & $\begin{array}{l}0.868 \% \mathrm{H} 2 / 0.868 \% \mathrm{CO} / 1.736 \% \mathrm{O} 2 / 96 . \\
529 \% \mathrm{Ar}\end{array}$ & 1140.0 & 15.9 & a00000243 & 20 & 238 & $5.87 \% \mathrm{H} 2 / 2.95 \% \mathrm{O} 2 / 91.18 \% \mathrm{Ar}$ & 964.0 & 4.1 & a00000634 & 35 \\
\hline 78 & 60/40\% $\mathrm{CO} / \mathrm{H} 2 /$ air & 1169.0 & 1.0 & $\mathrm{a} 00000286$ & 20 & 239 & $5.87 \% \mathrm{H} 2 / 2.95 \% \mathrm{O} 2 / 91.18 \% \mathrm{Ar}$ & 1002.0 & 4.0 & a00000635 & 25 \\
\hline 79 & $\begin{array}{l}1.7 \% \mathrm{H} 2 / 15.6 \% \mathrm{CO} / 17.5 \% \mathrm{O} 2 / 65.2 \% \\
\mathrm{Ar}\end{array}$ & 1033.0 & 1.1 & a00000299 & 20 & 240 & $5.87 \% \mathrm{H} 2 / 2.95 \% \mathrm{O} 2 / 91.18 \% \mathrm{Ar}$ & 1051.0 & 4.0 & a00000636 & 20 \\
\hline 80 & $\begin{array}{l}1.7 \% \mathrm{H} 2 / 15.6 \% \mathrm{CO} / 17.5 \% \mathrm{O} 2 / 65.2 \% \\
\mathrm{Ar}\end{array}$ & 991.0 & 2.6 & a00000301 & 20 & 241 & $5.87 \% \mathrm{H} 2 / 2.95 \% \mathrm{O} 2 / 91.18 \% \mathrm{Ar}$ & 1160.0 & 4.1 & a00000637 & 20 \\
\hline 81 & $\begin{array}{l}1.7 \% \mathrm{H} 2 / 15.6 \% \mathrm{CO} / 17.5 \% \mathrm{O} 2 / 65.2 \% \\
\mathrm{Ar}\end{array}$ & 1060.0 & 2.1 & a00000302 & 20 & 242 & $3.46 \% \mathrm{H} 2 / 3.49 \% \mathrm{O} 2 / 93.05 \% \mathrm{Ar}$ & 1018.0 & 15.4 & a00000638 & 35 \\
\hline 82 & $\begin{array}{l}1.7 \% \mathrm{H} 2 / 15.6 \% \mathrm{CO} / 17.5 \% \mathrm{O} 2 / 65.2 \% \\
\mathrm{Ar}\end{array}$ & 1148.0 & 2.3 & a00000303 & 20 & 243 & $3.46 \% \mathrm{H} 2 / 3.49 \% \mathrm{O} 2 / 93.05 \% \mathrm{Ar}$ & 1068.0 & 16.1 & a00000639 & 30 \\
\hline 83 & $\begin{array}{l}1.7 \% \mathrm{H} 2 / 15.6 \% \mathrm{CO} / 17.5 \% \mathrm{O} 2 / 65.2 \% \\
\mathrm{Ar}\end{array}$ & 1090.0 & 13.6 & a00000304 & 20 & 244 & $3.46 \% \mathrm{H} 2 / 3.49 \% \mathrm{O} 2 / 93.05 \% \mathrm{Ar}$ & 1109.0 & 16.0 & a00000640 & 25 \\
\hline 84 & $\begin{array}{l}1.7 \% \mathrm{H} 2 / 15.6 \% \mathrm{CO} / 17.5 \% \mathrm{O} 2 / 65.2 \% \\
\mathrm{Ar}\end{array}$ & 1158.0 & 14.7 & a00000305 & 20 & 245 & $3.46 \% \mathrm{H} 2 / 3.49 \% \mathrm{O} 2 / 93.05 \% \mathrm{Ar}$ & 1121.0 & 15.1 & a00000641 & 25 \\
\hline
\end{tabular}

11

American Institute of Aeronautics and Astronautics 


\begin{tabular}{|c|c|c|c|c|c|c|c|c|c|c|c|}
\hline$\#$ & Mixture & $\mathrm{T}_{5}, \mathrm{~K}$ & $\begin{array}{l}\mathrm{P}_{5} \\
\text { bar }\end{array}$ & $\begin{array}{l}\text { Attribute } \\
\text { ID }\end{array}$ & $\begin{array}{c}\text { Estim } \\
\text { ated } \\
\text { error, } \\
\%\end{array}$ & $\#$ & Mixture & $\mathrm{T}, \mathrm{K}$ & $\mathrm{p}$, bar & Attribute ID & $\begin{array}{c}\text { Estim } \\
\text { ated } \\
\text { error, } \\
\%\end{array}$ \\
\hline 85 & $\begin{array}{l}0.9 \% \mathrm{H} 2 / 16.5 \% \mathrm{CO} / 17.5 \% \mathrm{O} 2 / 65.2 \% \\
\mathrm{Ar}\end{array}$ & 968.0 & 1.2 & a00000307 & 20 & 246 & $5.87 \% \mathrm{H} 2 / 2.95 \% \mathrm{O} 2 / 91.18 \% \mathrm{Ar}$ & 1015.0 & 15.1 & a00000642 & 35 \\
\hline 86 & $\begin{array}{l}0.9 \% \mathrm{H} 2 / 16.5 \% \mathrm{CO} / 17.5 \% \mathrm{O} 2 / 65.2 \% \\
\mathrm{Ar}\end{array}$ & 1057.0 & 1.1 & a00000308 & 20 & 247 & $5.87 \% \mathrm{H} 2 / 2.95 \% \mathrm{O} 2 / 91.18 \% \mathrm{Ar}$ & 1098.0 & 15.1 & a00000643 & 25 \\
\hline 87 & $\begin{array}{l}0.9 \% \mathrm{H} 2 / 16.5 \% \mathrm{CO} / 17.5 \% \mathrm{O} 2 / 65.2 \% \\
\mathrm{Ar}\end{array}$ & 1263.0 & 1.1 & a00000309 & 20 & 248 & $5.87 \% \mathrm{H} 2 / 2.95 \% \mathrm{O} 2 / 91.18 \% \mathrm{Ar}$ & 1207.0 & 17.4 & a00000644 & 30 \\
\hline 88 & $\begin{array}{l}0.9 \% \mathrm{H} 2 / 16.5 \% \mathrm{CO} / 17.5 \% \mathrm{O} 2 / 65.2 \% \\
\mathrm{Ar}\end{array}$ & 977.0 & 2.3 & a00000310 & 20 & 249 & $5.87 \% \mathrm{H} 2 / 2.95 \% \mathrm{O} 2 / 91.18 \% \mathrm{Ar}$ & 1238.0 & 19.3 & a00000645 & 30 \\
\hline 89 & $\begin{array}{l}0.9 \% \mathrm{H} 2 / 16.5 \% \mathrm{CO} / 17.5 \% \mathrm{O} 2 / 65.2 \% \\
\mathrm{Ar}\end{array}$ & 1149.0 & 2.0 & a00000311 & 20 & 250 & $3.47 \% \mathrm{H} 2 / 3.47 \% \mathrm{O} 2 / 93.06 \% \mathrm{~N} 2$ & 932.0 & 0.9 & a00000646 & 40 \\
\hline 90 & $\begin{array}{l}0.9 \% \mathrm{H} 2 / 16.5 \% \mathrm{CO} / 17.5 \% \mathrm{O} 2 / 65.2 \% \\
\mathrm{Ar}\end{array}$ & 1304.0 & 1.7 & a00000312 & 20 & 251 & $3.47 \% \mathrm{H} 2 / 3.47 \% \mathrm{O} 2 / 93.06 \% \mathrm{~N} 2$ & 968.0 & 1.0 & a00000647 & 30 \\
\hline 91 & $\begin{array}{l}0.9 \% \mathrm{H} 2 / 16.5 \% \mathrm{CO} / 17.5 \% \mathrm{O} 2 / 65.2 \% \\
\mathrm{Ar}\end{array}$ & 1110.0 & 12.7 & a00000313 & 20 & 252 & $3.47 \% \mathrm{H} 2 / 3.47 \% \mathrm{O} 2 / 93.06 \% \mathrm{~N} 2$ & 992.0 & 1.0 & a00000648 & 30 \\
\hline 92 & \begin{tabular}{|l|}
$7.33 \% \mathrm{H} 2 / 9.71 \% \mathrm{CO} / 1.98 \% \mathrm{CO} 2 / 17.0$ \\
$1 \% \mathrm{O} 2 / 63.97 \% \mathrm{~N} 2$
\end{tabular} & 943.0 & 22.3 & a00000316 & 20 & 253 & $3.47 \% \mathrm{H} 2 / 3.47 \% \mathrm{O} 2 / 93.06 \% \mathrm{~N} 2$ & 1954.0 & 1.0 & a00000649 & 25 \\
\hline 93 & \begin{tabular}{|l|}
$7.33 \% \mathrm{H} 2 / 9.71 \% \mathrm{CO} / 1.98 \% \mathrm{CO} 2 / 17.0$ \\
$1 \% \mathrm{O} 2 / 63.97 \% \mathrm{~N} 2$
\end{tabular} & 1033.0 & 23.7 & a00000317 & 20 & 254 & $0.81 \% \mathrm{H} 2 / 4.03 \% \mathrm{O} 2 / 95.16 \% \mathrm{Ar}$ & 939.0 & 1.0 & a00000650 & 40 \\
\hline 94 & \begin{tabular}{|l|}
$7.33 \% \mathrm{H} 2 / 9.71 \% \mathrm{CO} / 1.98 \% \mathrm{CO} 2 / 17.0$ \\
$1 \% \mathrm{O} 2 / 63.97 \% \mathrm{~N} 2$
\end{tabular} & 1148.0 & 21.4 & a00000318 & 20 & 255 & $0.81 \% \mathrm{H} 2 / 4.03 \% \mathrm{O} 2 / 95.16 \% \mathrm{Ar}$ & 1026.0 & 1.0 & a00000651 & 25 \\
\hline 95 & $80 / 20 \% \mathrm{CO} / \mathrm{H} 2 /$ air & 909.0 & 1.2 & a00000322 & 30 & 256 & $0.81 \% \mathrm{H} 2 / 4.03 \% \mathrm{O} 2 / 95.16 \% \mathrm{Ar}$ & 1267.0 & 0.8 & a00000652 & 20 \\
\hline 96 & $80 / 20 \% \mathrm{CO} / \mathrm{H} 2 /$ air & 933.0 & 1.2 & a00000323 & 30 & 257 & $0.81 \% \mathrm{H} 2 / 4.03 \% \mathrm{O} 2 / 95.16 \% \mathrm{Ar}$ & 1670.0 & 0.9 & a00000653 & 25 \\
\hline 97 & $80 / 20 \% \mathrm{CO} / \mathrm{H} 2 /$ air & 947.0 & 1.2 & a00000324 & 30 & 258 & $0.81 \% \mathrm{H} 2 / 4.03 \% \mathrm{O} 2 / 95.16 \% \mathrm{Ar}$ & 2109.0 & 1.1 & a00000654 & 30 \\
\hline 98 & $0.8 \% \mathrm{H} 2 / 0.2 \% \mathrm{CO} / 1 \% \mathrm{O} 2 / 98 \% \mathrm{Ar}$ & 1299.0 & 12.0 & a00000334 & 20 & 259 & $4.03 \% \mathrm{H} 2 / 20.16 \% \mathrm{O} 2 / 75.81 \% \mathrm{Ar}$ & 889.0 & 1.0 & a00000655 & 35 \\
\hline 99 & $0.8 \% \mathrm{H} 2 / 0.2 \% \mathrm{CO} / 1 \% \mathrm{O} 2 / 98 \% \mathrm{Ar}$ & 1182.0 & 12.0 & a00000335 & 20 & 260 & $4.03 \% \mathrm{H} 2 / 20.16 \% \mathrm{O} 2 / 75.81 \% \mathrm{Ar}$ & 897.0 & 1.1 & a00000656 & 35 \\
\hline 100 & $0.8 \% \mathrm{H} 2 / 0.2 \% \mathrm{CO} / 1 \% \mathrm{O} 2 / 98 \% \mathrm{Ar}$ & 1096.0 & 12.0 & a00000336 & 20 & 261 & $4.03 \% \mathrm{H} 2 / 20.16 \% \mathrm{O} 2 / 75.81 \% \mathrm{Ar}$ & 975.0 & 1.1 & a00000657 & 25 \\
\hline 101 & $0.5 \% \mathrm{H} 2 / 0.5 \% \mathrm{CO} / 1 \% \mathrm{O} 2 / 98 \% \mathrm{Ar}$ & 1383.0 & 12.0 & a00000337 & 20 & 262 & $4.03 \% \mathrm{H} 2 / 20.16 \% \mathrm{O} 2 / 75.81 \% \mathrm{Ar}$ & 1154.0 & 0.7 & a00000658 & 20 \\
\hline 102 & $0.5 \% \mathrm{H} 2 / 0.5 \% \mathrm{CO} / 1 \% \mathrm{O} 2 / 98 \% \mathrm{Ar}$ & 1235.0 & 12.0 & a00000338 & 20 & 263 & $4.03 \% \mathrm{H} 2 / 20.16 \% \mathrm{O} 2 / 75.81 \% \mathrm{Ar}$ & 1675.0 & 1.1 & a00000659 & 30 \\
\hline 103 & $0.5 \% \mathrm{H} 2 / 0.5 \% \mathrm{CO} / 1 \% \mathrm{O} 2 / 98 \% \mathrm{Ar}$ & 1099.0 & 12.0 & a00000339 & 20 & 264 & $12.54 \% \mathrm{H} 2 / 1.57 \% \mathrm{O} 2 / 85.89 \% \mathrm{Ar}$ & 943.0 & 1.1 & a00000660 & 35 \\
\hline 104 & $0.1 \% \mathrm{H} 2 / 0.9 \% \mathrm{CO} / 1 \% \mathrm{O} 2 / 98 \% \mathrm{Ar}$ & 1387.0 & 12.0 & a00000340 & 20 & 265 & $12.54 \% \mathrm{H} 2 / 1.57 \% \mathrm{O} 2 / 85.89 \% \mathrm{Ar}$ & 953.0 & 0.9 & a00000661 & 30 \\
\hline 105 & $0.1 \% \mathrm{H} 2 / 0.9 \% \mathrm{CO} / 1 \% \mathrm{O} 2 / 98 \% \mathrm{Ar}$ & 1228.0 & 12.0 & a00000341 & 20 & 266 & $12.54 \% \mathrm{H} 2 / 1.57 \% \mathrm{O} 2 / 85.89 \% \mathrm{Ar}$ & 976.0 & 1.1 & a00000662 & 30 \\
\hline 106 & $0.1 \% \mathrm{H} 2 / 0.9 \% \mathrm{CO} / 1 \% \mathrm{O} 2 / 98 \% \mathrm{Ar}$ & 1116.0 & 12.0 & a00000342 & 20 & 267 & $12.54 \% \mathrm{H} 2 / 1.57 \% \mathrm{O} 2 / 85.89 \% \mathrm{Ar}$ & 1074.0 & 1.1 & a00000663 & 25 \\
\hline 107 & $0.8 \% \mathrm{H} 2 / 0.2 \% \mathrm{CO} / 1 \% \mathrm{O} 2 / 98 \% \mathrm{Ar}$ & 1264.0 & 32.0 & a00000343 & 20 & 268 & $12.54 \% \mathrm{H} 2 / 1.57 \% \mathrm{O} 2 / 85.89 \% \mathrm{Ar}$ & 1288.0 & 0.9 & a00000664 & 20 \\
\hline 108 & $0.8 \% \mathrm{H} 2 / 0.2 \% \mathrm{CO} / 1 \% \mathrm{O} 2 / 98 \% \mathrm{Ar}$ & 1243.0 & 32.0 & a00000344 & 20 & 269 & $12.54 \% \mathrm{H} 2 / 1.57 \% \mathrm{O} 2 / 85.89 \% \mathrm{Ar}$ & 2136.0 & 1.4 & a00000665 & 30 \\
\hline 109 & $0.8 \% \mathrm{H} 2 / 0.2 \% \mathrm{CO} / 1 \% \mathrm{O} 2 / 98 \% \mathrm{Ar}$ & 1185.0 & 32.0 & a00000345 & 20 & 270 & $3.47 \% \mathrm{H} 2 / 3.47 \% \mathrm{O} 2 / 93.06 \% \mathrm{~N} 2$ & 1006.0 & 3.9 & a00000666 & 30 \\
\hline 110 & $0.5 \% \mathrm{H} 2 / 0.5 \% \mathrm{CO} / 1 \% \mathrm{O} 2 / 98 \% \mathrm{Ar}$ & 1325.0 & 32.0 & a00000346 & 20 & 271 & $3.47 \% \mathrm{H} 2 / 3.47 \% \mathrm{O} 2 / 93.06 \% \mathrm{~N} 2$ & 1037.0 & 4.1 & a00000667 & 25 \\
\hline 111 & $0.5 \% \mathrm{H} 2 / 0.5 \% \mathrm{CO} / 1 \% \mathrm{O} 2 / 98 \% \mathrm{Ar}$ & 1204.0 & 32.0 & a00000347 & 20 & 272 & $3.47 \% \mathrm{H} 2 / 3.47 \% \mathrm{O} 2 / 93.06 \% \mathrm{~N} 2$ & 1060.0 & 4.2 & a00000668 & 25 \\
\hline 112 & $0.5 \% \mathrm{H} 2 / 0.5 \% \mathrm{CO} / 1 \% \mathrm{O} 2 / 98 \% \mathrm{Ar}$ & 1179.0 & 32.0 & a00000348 & 20 & 273 & $3.47 \% \mathrm{H} 2 / 3.47 \% \mathrm{O} 2 / 93.06 \% \mathrm{~N} 2$ & 1081.0 & 3.9 & a00000669 & 20 \\
\hline 113 & $0.1 \% \mathrm{H} 2 / 0.9 \% \mathrm{CO} / 1 \% \mathrm{O} 2 / 98 \% \mathrm{Ar}$ & 1327.0 & 32.0 & a00000349 & 20 & 274 & $3.47 \% \mathrm{H} 2 / 3.47 \% \mathrm{O} 2 / 93.06 \% \mathrm{~N} 2$ & 1257.0 & 3.9 & a00000670 & 20 \\
\hline 114 & $0.1 \% \mathrm{H} 2 / 0.9 \% \mathrm{CO} / 1 \% \mathrm{O} 2 / 98 \% \mathrm{Ar}$ & 1259.0 & 32.0 & a00000350 & 20 & 275 & $0.81 \% \mathrm{H} 2 / 4.03 \% \mathrm{O} 2 / 95.16 \% \mathrm{Ar}$ & 935.0 & 3.9 & a00000671 & 40 \\
\hline 115 & $0.1 \% \mathrm{H} 2 / 0.9 \% \mathrm{CO} / 1 \% \mathrm{O} 2 / 98 \% \mathrm{Ar}$ & 1166.0 & 32.0 & a00000351 & 20 & 276 & $0.81 \% \mathrm{H} 2 / 4.03 \% \mathrm{O} 2 / 95.16 \% \mathrm{Ar}$ & 986.0 & 3.9 & a00000672 & 30 \\
\hline 116 & $0.8 \% \mathrm{H} 2 / 0.2 \% \mathrm{CO} / 1 \% \mathrm{O} 2 / 98 \% \mathrm{Ar}$ & 1695.0 & 1.6 & a00000352 & 20 & 277 & $0.81 \% \mathrm{H} 2 / 4.03 \% \mathrm{O} 2 / 95.16 \% \mathrm{Ar}$ & 1075.0 & 3.5 & a00000673 & 20 \\
\hline 117 & $0.8 \% \mathrm{H} 2 / 0.2 \% \mathrm{CO} / 1 \% \mathrm{O} 2 / 98 \% \mathrm{Ar}$ & 1351.0 & 1.6 & $\mathrm{a} 00000353$ & 20 & 278 & $0.81 \% \mathrm{H} 2 / 4.03 \% \mathrm{O} 2 / 95.16 \% \mathrm{Ar}$ & 1360.0 & 4.2 & a00000674 & 25 \\
\hline 118 & $0.8 \% \mathrm{H} 2 / 0.2 \% \mathrm{CO} / 1 \% \mathrm{O} 2 / 98 \% \mathrm{Ar}$ & 980.0 & 1.6 & a00000354 & 20 & 279 & $4.03 \% \mathrm{H} 2 / 20.16 \% \mathrm{O} 2 / 75.81 \% \mathrm{Ar}$ & 932.0 & 4.1 & a00000675 & 40 \\
\hline 119 & $0.5 \% \mathrm{H} 2 / 0.5 \% \mathrm{CO} / 1 \% \mathrm{O} 2 / 98 \% \mathrm{Ar}$ & 2004.0 & 1.6 & $\mathrm{a} 00000355$ & 20 & 280 & $4.03 \% \mathrm{H} 2 / 20.16 \% \mathrm{O} 2 / 75.81 \% \mathrm{Ar}$ & 987.0 & 4.2 & $\mathrm{a} 00000676$ & 30 \\
\hline 120 & $0.5 \% \mathrm{H} 2 / 0.5 \% \mathrm{CO} / 1 \% \mathrm{O} 2 / 98 \% \mathrm{Ar}$ & 1273.0 & 1.6 & $\mathrm{a} 00000356$ & 20 & 281 & $4.03 \% \mathrm{H} 2 / 20.16 \% \mathrm{O} 2 / 75.81 \% \mathrm{Ar}$ & 1021.0 & 4.2 & a00000677 & 20 \\
\hline 121 & $0.5 \% \mathrm{H} 2 / 0.5 \% \mathrm{CO} / 1 \% \mathrm{O} 2 / 98 \% \mathrm{Ar}$ & 992.0 & 1.6 & $\mathrm{a} 00000357$ & 20 & 282 & $4.03 \% \mathrm{H} 2 / 20.16 \% \mathrm{O} 2 / 75.81 \% \mathrm{Ar}$ & 1131.0 & 4.1 & $\mathrm{a} 00000678$ & 25 \\
\hline 122 & $0.1 \% \mathrm{H} 2 / 0.9 \% \mathrm{CO} / 1 \% \mathrm{O} 2 / 98 \% \mathrm{Ar}$ & 1975.0 & 1.6 & $\mathrm{a} 00000358$ & 20 & 283 & $12.54 \% \mathrm{H} 2 / 1.57 \% \mathrm{O} 2 / 85.89 \% \mathrm{Ar}$ & 967.0 & 4.0 & a00000679 & 35 \\
\hline 123 & $0.1 \% \mathrm{H} 2 / 0.9 \% \mathrm{CO} / 1 \% \mathrm{O} 2 / 98 \% \mathrm{Ar}$ & 1436.0 & 1.6 & a00000359 & 20 & 284 & $12.54 \% \mathrm{H} 2 / 1.57 \% \mathrm{O} 2 / 85.89 \% \mathrm{Ar}$ & 997.0 & 4.1 & a00000680 & 30 \\
\hline 124 & $0.1 \% \mathrm{H} 2 / 0.9 \% \mathrm{CO} / 1 \% \mathrm{O} 2 / 98 \% \mathrm{Ar}$ & 1027.0 & 1.6 & a00000360 & 20 & 285 & $12.54 \% \mathrm{H} 2 / 1.57 \% \mathrm{O} 2 / 85.89 \% \mathrm{Ar}$ & 1017.0 & 4.2 & a00000681 & 20 \\
\hline 125 & $\begin{array}{l}0.444 \% \mathrm{H} 2 / 0.444 \% \mathrm{CO} / 0.223 \% \mathrm{H} 2 \mathrm{O} / 0 \\
.889 \% \mathrm{O} 2 / 98 \% \mathrm{Ar}\end{array}$ & 998.0 & 1.6 & a00000489 & 50 & 286 & $12.54 \% \mathrm{H} 2 / 1.57 \% \mathrm{O} 2 / 85.89 \% \mathrm{Ar}$ & 1463.0 & 4.3 & a00000682 & 25 \\
\hline 126 & $\begin{array}{l}0.444 \% \mathrm{H} 2 / 0.444 \% \mathrm{CO} / 0.223 \% \mathrm{H} 2 \mathrm{O} / 0 \\
.889 \% \mathrm{O} 2 / 98 \% \mathrm{Ar}\end{array}$ & 1146.0 & 1.6 & a00000490 & 30 & 287 & $3.47 \% \mathrm{H} 2 / 3.47 \% \mathrm{O} 2 / 93.06 \% \mathrm{~N} 2$ & 1060.0 & 15.7 & a00000683 & 35 \\
\hline 127 & $\begin{array}{l}8.91 \% \mathrm{H} 2 / 11.58 \% \mathrm{CO} / 10.25 \% \mathrm{O} 2 / 24.4 \\
4 \% \mathrm{CO} 2 / 44.83 \% \mathrm{~N} 2\end{array}$ & 981.0 & 1.2 & a00000491 & 20 & 288 & $3.47 \% \mathrm{H} 2 / 3.47 \% \mathrm{O} 2 / 93.06 \% \mathrm{~N} 2$ & 1165.0 & 15.9 & a00000684 & 25 \\
\hline 128 & $\begin{array}{l}8.91 \% \mathrm{H} 2 / 11.58 \% \mathrm{CO} / 10.25 \% \mathrm{O} 2 / 24.4 \\
4 \% \mathrm{CO} 2 / 44.83 \% \mathrm{~N} 2\end{array}$ & 1065.0 & 1.3 & a00000492 & 20 & 289 & $3.47 \% \mathrm{H} 2 / 3.47 \% \mathrm{O} 2 / 93.06 \% \mathrm{~N} 2$ & 1243.0 & 16.2 & a00000685 & 30 \\
\hline 129 & \begin{tabular}{|l|}
$0.444 \% \mathrm{H} 2 / 0.444 \% \mathrm{CO} / 0.223 \% \mathrm{H} 2 \mathrm{O} / 0$ \\
$.889 \% \mathrm{O} 2 / 98 \% \mathrm{Ar}$
\end{tabular} & 1786.0 & 1.6 & a00000493 & 30 & 290 & $0.81 \% \mathrm{H} 2 / 4.03 \% \mathrm{O} 2 / 95.16 \% \mathrm{Ar}$ & 1037.0 & 16.1 & a00000686 & 40 \\
\hline 130 & $\begin{array}{l}8.91 \% \mathrm{H} 2 / 11.58 \% \mathrm{CO} / 10.25 \% \mathrm{O} 2 / 24.4 \\
4 \% \mathrm{CO} 2 / 44.83 \% \mathrm{~N} 2\end{array}$ & 1135.0 & 1.2 & a00000494 & 20 & 291 & $0.81 \% \mathrm{H} 2 / 4.03 \% \mathrm{O} 2 / 95.16 \% \mathrm{Ar}$ & 1087.0 & 16.1 & a00000687 & 30 \\
\hline 131 & $\begin{array}{l}8.91 \% \mathrm{H} 2 / 11.58 \% \mathrm{CO} / 10.25 \% \mathrm{O} 2 / 24.4 \\
4 \% \mathrm{CO} 2 / 44.83 \% \mathrm{~N} 2\end{array}$ & 975.0 & 1.7 & a00000495 & 20 & 292 & $0.81 \% \mathrm{H} 2 / 4.03 \% \mathrm{O} 2 / 95.16 \% \mathrm{Ar}$ & 1141.0 & 16.3 & a00000688 & 25 \\
\hline 132 & $\begin{array}{l}8.91 \% \mathrm{H} 2 / 11.58 \% \mathrm{CO} / 10.25 \% \mathrm{O} 2 / 24.4 \\
4 \% \mathrm{CO} 2 / 44.83 \% \mathrm{~N} 2\end{array}$ & 999.0 & 1.8 & a00000496 & 20 & 293 & $0.81 \% \mathrm{H} 2 / 4.03 \% \mathrm{O} 2 / 95.16 \% \mathrm{Ar}$ & 1180.0 & 17.8 & a00000689 & 25 \\
\hline 133 & $\begin{array}{l}8.91 \% \mathrm{H} 2 / 11.58 \% \mathrm{CO} / 10.25 \% \mathrm{O} 2 / 24.4 \\
4 \% \mathrm{CO} 2 / 44.83 \% \mathrm{~N} 2\end{array}$ & 1048.0 & 1.7 & a00000497 & 20 & 294 & $0.81 \% \mathrm{H} 2 / 4.03 \% \mathrm{O} 2 / 95.16 \% \mathrm{Ar}$ & 1255.0 & 16.7 & a00000690 & 30 \\
\hline 134 & $\begin{array}{l}0.444 \% \mathrm{H} 2 / 0.444 \% \mathrm{CO} / 0.223 \% \mathrm{H} 2 \mathrm{O} / 0 \\
.889 \mathrm{O} 2 / 98 \% \mathrm{Ar}\end{array}$ & 1397.0 & 12.5 & a00000498 & 30 & 295 & $4.03 \% \mathrm{H} 2 / 20.16 \% \mathrm{O} 2 / 75.81 \% \mathrm{Ar}$ & 956.0 & 18.7 & a00000691 & 40 \\
\hline 135 & \begin{tabular}{|l|}
$0.444 \% \mathrm{H} 2 / 0.444 \% \mathrm{CO} / 0.223 \% \mathrm{H} 2 \mathrm{O} / 0$ \\
$.889 \mathrm{O} 2 / 98 \% \mathrm{Ar}$
\end{tabular} & 1284.0 & 12.5 & a00000499 & 30 & 296 & $4.03 \% \mathrm{H} 2 / 20.16 \% \mathrm{O} 2 / 75.81 \% \mathrm{Ar}$ & 1010.0 & 18.1 & a00000692 & 30 \\
\hline 136 & \begin{tabular}{|l|}
$0.444 \% \mathrm{H} 2 / 0.444 \% \mathrm{CO} / 0.223 \% \mathrm{H} 2 \mathrm{O} / 0$ \\
$.889 \mathrm{O} 2 / 98 \% \mathrm{Ar}$
\end{tabular} & 1100.0 & 12.5 & a00000500 & 30 & 297 & $4.03 \% \mathrm{H} 2 / 20.16 \% \mathrm{O} 2 / 75.81 \% \mathrm{Ar}$ & 1142.0 & 17.8 & a00000693 & 25 \\
\hline 137 & $\begin{array}{l}0.444 \% \mathrm{H} 2 / 0.444 \% \mathrm{CO} / 0.223 \% \mathrm{H} 2 \mathrm{O} / 0 \\
.889 \mathrm{O} 2 / 98 \% \mathrm{Ar}\end{array}$ & 1299.0 & 32.0 & a00000501 & 30 & 298 & $4.03 \% \mathrm{H} 2 / 20.16 \% \mathrm{O} 2 / 75.81 \% \mathrm{Ar}$ & 1178.0 & 17.6 & a00000694 & 30 \\
\hline 138 & $\begin{array}{l}0.444 \% \mathrm{H} 2 / 0.444 \% \mathrm{CO} / 0.223 \% \mathrm{H} 2 \mathrm{O} / 0 \\
.889 \mathrm{O} 2 / 98 \% \mathrm{Ar}\end{array}$ & 1186.0 & 32.0 & a00000502 & 30 & 299 & $12.54 \% \mathrm{H} 2 / 1.57 \% \mathrm{O} 2 / 85.89 \% \mathrm{Ar}$ & 947.0 & 15.1 & a00000695 & 45 \\
\hline 139 & $\begin{array}{l}0.46 \% \mathrm{H} 2 / 0.46 \% \mathrm{CO} / 0.15 \% \mathrm{CO} 2 / 0.39 \\
\% \mathrm{O} 2 / 98 \% \mathrm{Ar}\end{array}$ & 1883.0 & 1.6 & a00000503 & 30 & 300 & $12.54 \% \mathrm{H} 2 / 1.57 \% \mathrm{O} 2 / 85.89 \% \mathrm{Ar}$ & 1100.0 & 15.5 & a00000696 & 20 \\
\hline
\end{tabular}

12

American Institute of Aeronautics and Astronautics 


\begin{tabular}{|c|c|c|c|c|c|c|c|c|c|c|c|}
\hline \# & Mixture & $\mathrm{T}_{5}, \mathrm{~K}$ & $\begin{array}{l}\mathrm{P}_{5} \\
\text { bar }\end{array}$ & $\begin{array}{l}\text { Attribute } \\
\text { ID }\end{array}$ & $\begin{array}{c}\text { Estim } \\
\text { ated } \\
\text { error, } \\
\%\end{array}$ & \# & Mixture & $\mathrm{T}, \mathrm{K}$ & $\mathrm{p}$, bar & Attribute ID & $\begin{array}{c}\text { Estim } \\
\text { ated } \\
\text { error, } \\
\%\end{array}$ \\
\hline 140 & $\begin{array}{l}0.46 \% \mathrm{H} 2 / 0.46 \% \mathrm{CO} / 0.15 \% \mathrm{CO} 2 / 0.39 \\
\% \mathrm{O} 2 / 98 \% \mathrm{Ar}\end{array}$ & 1008.0 & 1.6 & a00000504 & 50 & 301 & $12.54 \% \mathrm{H} 2 / 1.57 \% \mathrm{O} 2 / 85.89 \% \mathrm{Ar}$ & 1227.0 & 14.9 & a00000697 & 25 \\
\hline 141 & $\begin{array}{l}0.46 \% \mathrm{H} 2 / 0.46 \% \mathrm{CO} / 0.15 \% \mathrm{CO} 2 / 0.39 \\
\% \mathrm{O} 2 / 98 \% \mathrm{Ar}\end{array}$ & 1360.0 & 12.5 & a00000505 & 30 & 302 & $5.917 \% \mathrm{H} 2 / 2.959 \% \mathrm{O} 2 / 91.124 \% \mathrm{Ar}$ & 1456.7 & 1.3 & a00000698 & 20 \\
\hline 142 & $\begin{array}{l}0.46 \% \mathrm{H} 2 / 0.46 \% \mathrm{CO} / 0.15 \% \mathrm{CO} 2 / 0.39 \\
\% \mathrm{O} 2 / 98 \% \mathrm{Ar}\end{array}$ & 1122.0 & 12.5 & a00000506 & 30 & 303 & $5.917 \% \mathrm{H} 2 / 2.959 \% \mathrm{O} 2 / 91.124 \% \mathrm{Ar}$ & 1163.2 & 1.2 & a00000699 & 20 \\
\hline 143 & $\begin{array}{l}0.46 \% \mathrm{H} 2 / 0.46 \% \mathrm{CO} / 0.15 \% \mathrm{CO} 2 / 0.39 \\
\% \mathrm{O} 2 / 98 \% \mathrm{Ar}\end{array}$ & 1291.0 & 32.0 & a00000507 & 30 & 304 & $5.917 \% \mathrm{H} 2 / 2.959 \% \mathrm{O} 2 / 91.124 \% \mathrm{Ar}$ & 915.1 & 1.2 & a00000700 & 25 \\
\hline 144 & $\begin{array}{l}0.46 \% \mathrm{H} 2 / 0.46 \% \mathrm{CO} / 0.15 \% \mathrm{CO} 2 / 0.39 \\
\% \mathrm{O} 2 / 98 \% \mathrm{Ar}\end{array}$ & 1233.0 & 32.0 & a00000508 & 30 & 305 & $3.41 \% \mathrm{H} 2 / 3.41 \% \mathrm{O} 2 / 93.058 \% \mathrm{Ar}$ & 1193.6 & 5.0 & $\mathrm{a} 00000701$ & 25 \\
\hline 145 & $\begin{array}{l}0.46 \% \mathrm{H} 2 / 0.46 \% \mathrm{CO} / 0.15 \% \mathrm{CO} 2 / 0.39 \\
\% \mathrm{O} 2 / 98 \% \mathrm{Ar}\end{array}$ & 1150.0 & 32.0 & a00000509 & 30 & 306 & $3.41 \% \mathrm{H} 2 / 3.41 \% \mathrm{O} 2 / 93.058 \% \mathrm{Ar}$ & 1050.4 & 5.0 & a00000702 & 25 \\
\hline 146 & $0.5 \% \mathrm{H} 2 / 0.5 \% \mathrm{CO} / 1 \% \mathrm{O} 2 / 98 \% \mathrm{Ar}$ & 1763.0 & 1.6 & a00000538 & 30 & 307 & $3.41 \% \mathrm{H} 2 / 3.41 \% \mathrm{O} 2 / 93.058 \% \mathrm{Ar}$ & 1022.1 & 5.0 & a00000703 & 30 \\
\hline 147 & $0.5 \% \mathrm{H} 2 / 0.5 \% \mathrm{CO} / 1 \% \mathrm{O} 2 / 98 \% \mathrm{Ar}$ & 1503.0 & 1.6 & a00000539 & 25 & 308 & $3.41 \% \mathrm{H} 2 / 3.41 \% \mathrm{O} 2 / 93.058 \% \mathrm{Ar}$ & 1222.1 & 10.0 & $\mathrm{a} 00000704$ & 30 \\
\hline 148 & $0.5 \% \mathrm{H} 2 / 0.5 \% \mathrm{CO} / 1 \% \mathrm{O} 2 / 98 \% \mathrm{Ar}$ & 1068.0 & 1.6 & $\mathrm{a} 00000540$ & 25 & 309 & $3.41 \% \mathrm{H} 2 / 3.41 \% \mathrm{O} 2 / 93.058 \% \mathrm{Ar}$ & 1122.3 & 10.0 & $\mathrm{a} 00000705$ & 25 \\
\hline 149 & $\begin{array}{l}0.46 \% \mathrm{H} 2 / 0.46 \% \mathrm{CO} / 0.15 \% \mathrm{CO} 2 / 0.39 \\
\% \mathrm{O} 2 / 98 \% \mathrm{Ar}\end{array}$ & 1367.0 & 1.6 & $\mathrm{a} 00000542$ & 30 & 310 & $3.41 \% \mathrm{H} 2 / 3.41 \% \mathrm{O} 2 / 93.058 \% \mathrm{Ar}$ & 1077.2 & 10.0 & a00000706 & 25 \\
\hline 150 & $\begin{array}{l}0.46 \% \mathrm{H} 2 / 0.46 \% \mathrm{CO} / 0.15 \% \mathrm{CO} 2 / 0.39 \\
\% \mathrm{O} 2 / 98 \% \mathrm{Ar}\end{array}$ & 1199.0 & 12.5 & a00000543 & 30 & 311 & $3.41 \% \mathrm{H} 2 / 3.41 \% \mathrm{O} 2 / 93.058 \% \mathrm{Ar}$ & 1041.5 & 10.0 & $\mathrm{a} 00000707$ & 35 \\
\hline 151 & $\begin{array}{l}8.91 \% \mathrm{H} 2 / 11.58 \% \mathrm{CO} / 10.25 \% \mathrm{O} 2 / 24.4 \\
4 \% \mathrm{CO} 2 / 44.83 \% \mathrm{~N} 2\end{array}$ & 1017.0 & 1.9 & $\mathrm{a} 00000547$ & 50 & 312 & $3.41 \% \mathrm{H} 2 / 3.41 \% \mathrm{O} 2 / 93.058 \% \mathrm{Ar}$ & 1036.1 & 10.0 & a00000708 & 30 \\
\hline 152 & $\begin{array}{l}8.91 \% \mathrm{H} 2 / 11.58 \% \mathrm{CO} / 10.25 \% \mathrm{O} 2 / 24.4 \\
4 \% \mathrm{CO} 2 / 44.83 \% \mathrm{~N} 2\end{array}$ & 1024.0 & 2.6 & a00000548 & 50 & 313 & $3.41 \% \mathrm{H} 2 / 3.41 \% \mathrm{O} 2 / 93.058 \% \mathrm{Ar}$ & 1267.2 & 20.0 & a00000709 & 35 \\
\hline 153 & $\begin{array}{l}8.91 \% \mathrm{H} 2 / 11.58 \% \mathrm{CO} / 10.25 \% \mathrm{O} 2 / 24.4 \\
4 \% \mathrm{CO} 2 / 44.83 \% \mathrm{~N} 2\end{array}$ & 1054.0 & 2.4 & a00000549 & 30 & 314 & $3.41 \% \mathrm{H} 2 / 3.41 \% \mathrm{O} 2 / 93.058 \% \mathrm{Ar}$ & 1168.8 & 20.0 & a00000710 & 30 \\
\hline 154 & $\begin{array}{l}8.91 \% \mathrm{H} 2 / 11.58 \% \mathrm{CO} / 10.25 \% \mathrm{O} 2 / 24.4 \\
4 \% \mathrm{CO} 2 / 44.83 \% \mathrm{~N} 2\end{array}$ & 1091.0 & 2.3 & $\mathrm{a} 00000550$ & 30 & 315 & $3.41 \% \mathrm{H} 2 / 3.41 \% \mathrm{O} 2 / 93.058 \% \mathrm{Ar}$ & 1092.7 & 20.0 & a00000711 & 35 \\
\hline 155 & $2.81 \% \mathrm{H} 2 / 1.19 \% \mathrm{CO} / 2 \% \mathrm{O} 2 / 94 \% \mathrm{Ar}$ & 1161.2 & 1.5 & a00000551 & 35 & 316 & $3.41 \% \mathrm{H} 2 / 3.41 \% \mathrm{O} 2 / 93.058 \% \mathrm{Ar}$ & 1009.4 & 20.0 & $\mathrm{a} 00000712$ & 40 \\
\hline 156 & $2.81 \% \mathrm{H} 2 / 1.19 \% \mathrm{CO} / 2 \% \mathrm{O} 2 / 94 \% \mathrm{Ar}$ & 1025.0 & 1.7 & $\mathrm{a} 00000552$ & 25 & 317 & $30 \% \mathrm{H} 2 / 14.8 \% \mathrm{O} 2 / 55.2 \% \mathrm{~N} 2$ & 1179.7 & 2.0 & $\mathrm{a} 00000713$ & 35 \\
\hline 157 & $2.81 \% \mathrm{H} 2 / 1.19 \% \mathrm{CO} / 2 \% \mathrm{O} 2 / 94 \% \mathrm{Ar}$ & 978.5 & 1.7 & $\mathrm{a} 00000553$ & 40 & 318 & $30 \% \mathrm{H} 2 / 14.8 \% \mathrm{O} 2 / 55.2 \% \mathrm{~N} 2$ & 1077.3 & 2.0 & a00000714 & 35 \\
\hline 158 & $2.81 \% \mathrm{H} 2 / 1.19 \% \mathrm{CO} / 2 \% \mathrm{O} 2 / 94 \% \mathrm{Ar}$ & 944.8 & 1.9 & a00000554 & 50 & 319 & $30 \% \mathrm{H} 2 / 14.8 \% \mathrm{O} 2 / 55.2 \% \mathrm{~N} 2$ & 1025.8 & 2.0 & a00000715 & 30 \\
\hline 159 & $2.81 \% \mathrm{H} 2 / 1.19 \% \mathrm{CO} / 2 \% \mathrm{O} 2 / 94 \% \mathrm{Ar}$ & 932.9 & 1.9 & $\mathrm{a} 00000555$ & 45 & 320 & $30 \% \mathrm{H} 2 / 14.8 \% \mathrm{O} 2 / 55.2 \% \mathrm{~N} 2$ & 994.4 & 2.0 & a00000716 & 35 \\
\hline 160 & $2.81 \% \mathrm{H} 2 / 1.19 \% \mathrm{CO} / 2 \% \mathrm{O} 2 / 94 \% \mathrm{Ar}$ & 902.1 & 1.8 & $\mathrm{a} 00000556$ & 90 & 321 & $30 \% \mathrm{H} 2 / 14.8 \% \mathrm{O} 2 / 55.2 \% \mathrm{~N} 2$ & 986.6 & 2.0 & $\mathrm{a} 00000717$ & 35 \\
\hline 161 & $2.81 \% \mathrm{H} 2 / 1.19 \% \mathrm{CO} / 2 \% \mathrm{O} 2 / 94 \% \mathrm{Ar}$ & 1178.8 & 9.1 & $\mathrm{a} 00000557$ & 25 & & & & & & \\
\hline
\end{tabular}

Appendix 2. Laminar flame speed QoIs

\begin{tabular}{|c|c|c|c|c|c|c|c|c|c|c|c|}
\hline \# & Mixture & $\mathrm{T}_{0}, \mathrm{~K}$ & $\begin{array}{l}\text { P, } \\
\text { bar }\end{array}$ & $\varphi$ & $\begin{array}{c}\text { Estim } \\
\text { ated } \\
\text { error, } \\
\%\end{array}$ & \# & Mixture & $\mathrm{T}_{0}, \mathrm{~K}$ & $\begin{array}{l}\text { P, } \\
\text { bar }\end{array}$ & $\varphi$ & $\begin{array}{c}\text { Estim } \\
\text { ated } \\
\text { error, } \\
\%\end{array}$ \\
\hline 1 & 50/50\% CO/H2/air & 300 & 1 & 0.8 & 10 & 80 & $85 \% \mathrm{H} 2-15 \% \mathrm{CO} / 11 \% \mathrm{O} 2-89 \% \mathrm{He}$ & 298 & 1 & 0.6 & 10 \\
\hline 2 & $50 / 50 \% \mathrm{CO} / \mathrm{H} 2 /$ air & 300 & 1 & 1.2 & 10 & 81 & $85 \% \mathrm{H} 2-15 \% \mathrm{CO} / 11 \% \mathrm{O} 2-89 \% \mathrm{He}$ & 298 & 5 & 0.6 & 10 \\
\hline 3 & $50 / 50 \% \mathrm{CO} / \mathrm{H} 2 /$ air & 300 & 1 & 2.5 & 15 & 82 & $\begin{array}{l}75 / 25 \% \mathrm{CO} / \mathrm{H} 2 / \mathrm{He}, \mathrm{O} 2 / \mathrm{He}=1: 6, \\
10 \% \text { dil. with } \mathrm{CO} 2\end{array}$ & 298 & 5 & 0.8 & 10 \\
\hline 4 & $50 / 50 \% \mathrm{CO} / \mathrm{H} 2 /$ air & 300 & 1 & 4 & 20 & 83 & $\begin{array}{l}75 / 25 \% \mathrm{CO} / \mathrm{H} 2 / \mathrm{He}, \mathrm{O} 2 / \mathrm{He}=1: 6, \\
30 \% \text { dil. with } \mathrm{CO} 2\end{array}$ & 298 & 5 & 0.8 & 10 \\
\hline 5 & $50 / 50 \% \mathrm{CO} / \mathrm{H} 2 /$ air & 300 & 2 & 0.6 & 20 & 84 & $\begin{array}{l}75 / 25 \% \mathrm{CO} / \mathrm{H} 2 / \mathrm{He}, \mathrm{O} 2 / \mathrm{He}=1: 6 \text {, } \\
10 \% \text { dil. with } \mathrm{CO} 2\end{array}$ & 298 & 10 & 0.8 & 10 \\
\hline 6 & $50 / 50 \% \mathrm{CO} / \mathrm{H} 2 /$ air & 300 & 2 & 1.4 & 10 & 85 & $\begin{array}{l}75 / 25 \% \mathrm{CO} / \mathrm{H} 2 / \mathrm{He}, \mathrm{O} 2 / \mathrm{He}=1: 6 \text {, } \\
30 \% \text { dil. with } \mathrm{CO} 2\end{array}$ & 298 & 10 & 0.8 & 10 \\
\hline 7 & 50/50\% CO/H2/air & 300 & 2 & 2 & 10 & 86 & $40 \% / 40 \% / 20 \% \mathrm{CO} / \mathrm{H} 2 / \mathrm{CO} 2$ & 295 & 1 & 0.6 & 10 \\
\hline 8 & $50 / 50 \% \mathrm{CO} / \mathrm{H} 2 /$ air & 300 & 2 & 4 & 20 & 87 & $40 \% / 40 \% / 20 \% \mathrm{CO} / \mathrm{H} 2 / \mathrm{CO} 2$ & 295 & 1 & 1.4 & 10 \\
\hline 9 & $95 / 5 \% \mathrm{CO} / \mathrm{H} 2 / \mathrm{He}$ & 300 & 5 & 1.2 & 10 & 88 & $40 \% / 40 \% / 20 \% \mathrm{CO} / \mathrm{H} 2 / \mathrm{CO} 2$ & 295 & 1 & 1.8 & 10 \\
\hline 10 & $95 / 5 \% \mathrm{CO} / \mathrm{H} 2 / \mathrm{He}$ & 300 & 5 & 1.4 & 10 & 89 & $40 \% / 40 \% / 20 \% \mathrm{CO} / \mathrm{H} 2 / \mathrm{CO} 2$ & 295 & 1 & 2.2 & 15 \\
\hline 11 & $95 / 5 \% \mathrm{CO} / \mathrm{H} 2 / \mathrm{He}$ & 300 & 5 & 2 & 15 & 90 & $\begin{array}{l}\text { 70/30\% CO/H2/air, } 10 \% \text { dil. with } \\
\mathrm{CO} 2\end{array}$ & 295 & 1 & 1 & 10 \\
\hline 12 & $95 / 5 \% \mathrm{CO} / \mathrm{H} 2 / \mathrm{He}$ & 300 & 5 & 3.5 & 20 & 91 & $\begin{array}{l}\text { 70/30\% CO/H2/air, } 60 \% \text { dil. with } \\
\mathrm{CO} 2\end{array}$ & 295 & 1 & 1 & 10 \\
\hline 13 & $95 / 5 \% \mathrm{CO} / \mathrm{H} 2 / \mathrm{He}$ & 300 & 10 & 0.75 & 15 & 92 & $\begin{array}{l}\text { 70/30\% CO/H2/air, } 10 \% \text { dil. with } \\
\mathrm{CO} 2\end{array}$ & 295 & 1 & 0.6 & 10 \\
\hline 14 & $95 / 5 \% \mathrm{CO} / \mathrm{H} 2 / \mathrm{He}$ & 300 & 10 & 1.2 & 15 & 93 & $\begin{array}{l}\text { 70/30\% CO/H2/air, } 60 \% \text { dil. with } \\
\mathrm{CO} 2\end{array}$ & 295 & 1 & 0.6 & 10 \\
\hline 15 & $95 / 5 \% \mathrm{CO} / \mathrm{H} 2 / \mathrm{He}$ & 300 & 10 & 1.4 & 15 & 94 & $\begin{array}{l}30 / 70 \% \mathrm{CO} / \mathrm{H} 2 / \text { air, } 10 \% \text { dil. with } \\
\mathrm{CO} 2\end{array}$ & 295 & 1 & 1 & 10 \\
\hline 16 & $95 / 5 \% \mathrm{CO} / \mathrm{H} 2 / \mathrm{He}$ & 300 & 10 & 1.8 & 15 & 95 & $\begin{array}{l}30 / 70 \% \mathrm{CO} / \mathrm{H} 2 / \text { air, } 60 \% \text { dil. with } \\
\mathrm{CO} 2\end{array}$ & 295 & 1 & 1 & 10 \\
\hline 17 & $95 / 5 \% \mathrm{CO} / \mathrm{H} 2 / \mathrm{He}$ & 300 & 10 & 3.5 & 25 & 96 & $\begin{array}{l}30 / 70 \% \mathrm{CO} / \mathrm{H} 2 / \text { air, } 10 \% \text { dil. with } \\
\mathrm{CO} 2\end{array}$ & 295 & 1 & 0.6 & 10 \\
\hline 18 & $95 / 5 \% \mathrm{CO} / \mathrm{H} 2 / \mathrm{He}$ & 300 & 20 & 1 & 20 & 97 & $\begin{array}{l}30 / 70 \% \mathrm{CO} / \mathrm{H} 2 / \text { air, } 60 \% \text { dil. with } \\
\mathrm{CO} 2\end{array}$ & 295 & 1 & 0.6 & 10 \\
\hline 19 & $95 / 5 \% \mathrm{CO} / \mathrm{H} 2 / \mathrm{He}$ & 300 & 20 & 1.4 & 20 & 98 & $\begin{array}{l}\text { 50/50\% CO/H2/air, } 10 \% \text { dil. with } \\
\mathrm{CO} 2\end{array}$ & 295 & 1 & 1 & 10 \\
\hline 20 & $95 / 5 \% \mathrm{CO} / \mathrm{H} 2 / \mathrm{He}$ & 300 & 20 & 2 & 20 & 99 & $\begin{array}{l}50 / 50 \% \mathrm{CO} / \mathrm{H} 2 / \text { air, } 60 \% \text { dil. with } \\
\mathrm{CO} 2\end{array}$ & 295 & 1 & 1 & 10 \\
\hline 21 & $95 / 5 \% \mathrm{CO} / \mathrm{H} 2 / \mathrm{He}$ & 300 & 20 & 3.5 & 25 & 100 & $50 \% \mathrm{H} 2-50 \% \mathrm{CO} / \mathrm{O} 2-47 \% \mathrm{H} 2 \mathrm{O}$ & 400 & 1 & 0.6 & 10 \\
\hline 22 & $95 / 5 \% \mathrm{CO} / \mathrm{H} 2 / \mathrm{He}$ & 300 & 40 & 1.2 & 20 & 101 & $50 \% \mathrm{H} 2-50 \% \mathrm{CO} / \mathrm{O} 2-47 \% \mathrm{H} 2 \mathrm{O}$ & 400 & 1 & 1.2 & 10 \\
\hline
\end{tabular}

13

American Institute of Aeronautics and Astronautics 


\begin{tabular}{|c|c|c|c|c|c|c|c|c|c|c|c|}
\hline$\#$ & Mixture & $\mathrm{T}_{0}, \mathrm{~K}$ & $\begin{array}{l}\mathrm{P}, \\
\text { bar }\end{array}$ & $\varphi$ & $\begin{array}{c}\text { Estim } \\
\text { ated } \\
\text { error, } \\
\%\end{array}$ & $\#$ & Mixture & $\mathrm{T}_{0}, \mathrm{~K}$ & $\begin{array}{l}\mathrm{P}, \\
\text { bar }\end{array}$ & $\varphi$ & $\begin{array}{c}\text { Estim } \\
\text { ated } \\
\text { error, } \\
\%\end{array}$ \\
\hline 23 & $95 / 5 \% \mathrm{CO} / \mathrm{H} 2 / \mathrm{He}$ & 300 & 40 & 1.4 & 20 & 102 & $50 \% \mathrm{H} 2-50 \% \mathrm{CO} / \mathrm{O} 2-47 \% \mathrm{H} 2 \mathrm{O}$ & 400 & 1 & 2 & 10 \\
\hline 24 & $95 / 5 \% \mathrm{CO} / \mathrm{H} 2 / \mathrm{He}$ & 300 & 40 & 2 & 20 & 103 & 95/5\% CO/H2/air & 298 & 1 & 2 & 10 \\
\hline 25 & $95 / 5 \% \mathrm{CO} / \mathrm{H} 2 / \mathrm{He}$ & 300 & 40 & 3 & 25 & 104 & 95/5\% CO/H2/air & 298 & 1 & 2.4 & 15 \\
\hline 26 & 95/5\% CO/H2/air & 300 & 1 & 0.6 & 15 & 105 & $\begin{array}{l}50 / 50 \% \mathrm{CO} / \mathrm{H} 2 / \text { air diluted with } \\
20 \% \mathrm{~N} 2\end{array}$ & 298 & 1 & 1 & 10 \\
\hline 27 & 95/5\% CO/H2/air & 300 & 1 & 1 & 20 & 106 & $\begin{array}{l}50 / 50 \% \mathrm{CO} / \mathrm{H} 2 / \text { air diluted with } \\
20 \% \mathrm{~N} 2\end{array}$ & 298 & 1 & 1.8 & 10 \\
\hline 28 & $95 / 5 \% \mathrm{CO} / \mathrm{H} 2 / \mathrm{air}$ & 300 & 1 & 1.5 & 20 & 107 & $\begin{array}{l}50 / 50 \% \mathrm{CO} / \mathrm{H} 2 / \text { air diluted with } \\
20 \% \mathrm{~N} 2\end{array}$ & 298 & 1 & 4.2 & 20 \\
\hline 29 & 95/5\% CO/H2/air & 300 & 1 & 2.5 & 15 & 108 & $\begin{array}{l}\text { 50/50\% CO/H2/air diluted with } \\
60 \% \mathrm{~N} 2\end{array}$ & 298 & 1 & 0.8 & 10 \\
\hline 30 & 95/5\% CO/H2/air & 300 & 1 & 4 & 20 & 109 & $\begin{array}{l}50 / 50 \% \mathrm{CO} / \mathrm{H} 2 / \text { air diluted with } \\
60 \% \mathrm{~N} 2\end{array}$ & 298 & 1 & 1.4 & 10 \\
\hline 31 & 95/5\% CO/H2/air & 300 & 1 & 6 & 20 & 110 & $\begin{array}{l}50 / 50 \% \mathrm{CO} / \mathrm{H} 2 / \text { air diluted with } \\
60 \% \mathrm{~N} 2\end{array}$ & 298 & 1 & 2.2 & 15 \\
\hline 33 & $95 / 5 \% \mathrm{CO} / \mathrm{H} 2 /$ air & 300 & 2 & 2.5 & 15 & 112 & $\begin{array}{l}5 \% \mathrm{H} 2-95 \% \mathrm{CO}, \mathrm{O} 2,30 \% \text { dil. with } \\
\mathrm{N} 2\end{array}$ & 298 & 1 & 1.2 & 10 \\
\hline 34 & 95/5\% CO/H2/air & 300 & 2 & 4 & 20 & 113 & $\begin{array}{l}5 \% \mathrm{H} 2-95 \% \mathrm{CO}, \mathrm{O} 2,30 \% \text { dil. with } \\
\mathrm{N} 2\end{array}$ & 298 & 1 & 2 & 10 \\
\hline 35 & $50 / 50 \% \mathrm{CO} / \mathrm{H} 2 / \mathrm{He}$ & 300 & 5 & 1 & 10 & 114 & $\begin{array}{l}50 \% \mathrm{H} 2-50 \% \mathrm{CO}, \mathrm{O} 2,50 \% \text { dil. with } \\
\mathrm{N} 2\end{array}$ & 298 & 1 & 0.6 & 10 \\
\hline 36 & $50 / 50 \% \mathrm{CO} / \mathrm{H} 2 / \mathrm{He}$ & 300 & 5 & 1.8 & 10 & 115 & $\begin{array}{l}50 \% \mathrm{H} 2-50 \% \mathrm{CO}, \mathrm{O} 2,50 \% \text { dil. with } \\
\mathrm{N} 2\end{array}$ & 298 & 1 & 1.2 & 10 \\
\hline 37 & $50 / 50 \% \mathrm{CO} / \mathrm{H} 2 / \mathrm{He}$ & 300 & 5 & 3.5 & 20 & 116 & $\begin{array}{l}50 \% \mathrm{H} 2-50 \% \mathrm{CO}, \mathrm{O} 2,50 \% \text { dil. with } \\
\mathrm{N} 2\end{array}$ & 298 & 1 & 2 & 10 \\
\hline 38 & $50 / 50 \% \mathrm{CO} / \mathrm{H} 2 / \mathrm{He}$ & 300 & 10 & 1 & 15 & 117 & $95 \% \mathrm{CO}-5 \% \mathrm{H} 2 /$ air/H2O, H2O=20\% & 373 & 1 & 1.2 & 10 \\
\hline 39 & $50 / 50 \% \mathrm{CO} / \mathrm{H} 2 / \mathrm{He}$ & 300 & 10 & 1.8 & 15 & 118 & $95 \% / 5 \% \mathrm{CO} / \mathrm{H} 2 /$ air $\mathrm{ZH} 2 \mathrm{O}=20 \%$ & 373 & 1 & 2 & 10 \\
\hline 40 & $50 / 50 \% \mathrm{CO} / \mathrm{H} 2 / \mathrm{He}$ & 300 & 10 & 3.5 & 25 & 119 & $95 \% / 5 \% \mathrm{CO} / \mathrm{H} 2 /$ air $\mathrm{ZH} 2 \mathrm{O}=20 \%$ & 373 & 1 & 3 & 15 \\
\hline 41 & 95/5\% CO/H2/air & 300 & 0.5 & 0.8 & 10 & 120 & $50 \% / 50 \% \mathrm{CO} / \mathrm{H} 2 /$ air $\mathrm{ZH} 2 \mathrm{O}=20 \%$ & 373 & 5 & 0.8 & 10 \\
\hline 42 & 95/5\% CO/H2/air & 300 & 0.5 & 1 & 10 & 121 & $50 \% / 50 \% \mathrm{CO} / \mathrm{H} 2 /$ air $\mathrm{ZH} 2 \mathrm{O}=20 \%$ & 373 & 5 & 1.5 & 10 \\
\hline 43 & $95 / 5 \% \mathrm{CO} / \mathrm{H} 2 /$ air & 300 & 0.5 & 1.6 & 10 & 122 & $\begin{array}{l}50 \% \mathrm{CO}-50 \% \mathrm{H} 2 / \mathrm{air} / \mathrm{H} 2 \mathrm{O} \\
\mathrm{H} 2 \mathrm{O}=20 \%\end{array}$ & 373 & 5 & 2.5 & 15 \\
\hline 46 & 95/5\% CO/H2/air & 300 & 4 & 0.6 & 10 & 125 & $50 \% \mathrm{H} 2-50 \% \mathrm{CO} /$ air, $\mathrm{CO} 2=0.15$ & 373 & 1 & 3 & 15 \\
\hline 47 & 95/5\% CO/H2/air & 300 & 4 & 1.6 & 10 & 126 & $50 \% \mathrm{H} 2-50 \% \mathrm{CO} /$ air, $\mathrm{H} 2 \mathrm{O}=0.15$ & 373 & 1 & 0.8 & 10 \\
\hline 48 & 95/5\% CO/H2/air & 300 & 4 & 2.4 & 15 & 127 & $50 \% \mathrm{H} 2-50 \% \mathrm{CO} /$ air, $\mathrm{H} 2 \mathrm{O}=0.15$ & 373 & 1 & 2 & 10 \\
\hline 49 & 95/5\% CO/H2/air & 300 & 4 & 5 & 20 & 128 & $50 \% \mathrm{H} 2-50 \% \mathrm{CO} /$ air, $\mathrm{H} 2 \mathrm{O}=0.15$ & 373 & 1 & 3 & 15 \\
\hline 50 & $50 / 50 \% \mathrm{CO} / \mathrm{H} 2 /$ air & 500 & 1 & 0.63 & 10 & 129 & H2/air & 298 & 1 & 0.5 & 10 \\
\hline 51 & 50/50\% CO/H2/air & 500 & 1 & 1.01 & 10 & 130 & H2/air & 298 & 1 & 1.5 & 10 \\
\hline 52 & $50 / 50 \% \mathrm{CO} / \mathrm{H} 2 /$ air & 700 & 1 & 0.63 & 10 & 131 & H2/air & 298 & 1 & 4.5 & 20 \\
\hline 53 & $50 / 50 \% \mathrm{CO} / \mathrm{H} 2 /$ air & 700 & 1 & 0.73 & 10 & 132 & $\mathrm{H} 2 / \mathrm{O} 2 / \mathrm{Ar}, \mathrm{O} 2 /(\mathrm{O} 2+\mathrm{Ar})=21 \%$ & 298 & 1 & 0.6 & 10 \\
\hline 54 & 50/50\% CO/H2/air & 700 & 1 & 0.9 & 10 & 133 & $\mathrm{H} 2 / \mathrm{O} 2 / \mathrm{Ar}, \mathrm{O} 2 /(\mathrm{O} 2+\mathrm{Ar})=21 \%$ & 298 & 1 & 1.8 & 10 \\
\hline 55 & $80 / 20 \% \mathrm{CO} / \mathrm{H} 2 / \mathrm{He}$ & 600 & 15 & 0.6 & 20 & 134 & $\mathrm{H} 2 / \mathrm{O} 2 / \mathrm{Ar}, \mathrm{O} 2 /(\mathrm{O} 2+\mathrm{Ar})=21 \%$ & 298 & 1 & 4.5 & 20 \\
\hline 56 & $50 / 50 \% \mathrm{CO} / \mathrm{H} 2 / \mathrm{He}$ & 600 & 15 & 0.6 & 20 & 135 & $\mathrm{H} 2 / \mathrm{O} 2 / \mathrm{He}, \mathrm{O} 2 /(\mathrm{O} 2+\mathrm{He})=21 \%$ & 298 & 1 & 0.6 & 10 \\
\hline 57 & $20 / 80 \% \mathrm{CO} / \mathrm{H} 2 / \mathrm{He}$ & 600 & 15 & 0.6 & 20 & 136 & $\mathrm{H} 2 / \mathrm{O} 2 / \mathrm{He}, \mathrm{O} 2 /(\mathrm{O} 2+\mathrm{He})=21 \%$ & 298 & 1 & 1.8 & 10 \\
\hline 58 & $\begin{array}{l}\text { 95/5\% CO/H2/air, } 0 \% \text { dil. with } \\
\mathrm{H} 20\end{array}$ & 323 & 1 & 0.6 & 10 & 137 & $\mathrm{H} 2 / \mathrm{O} 2 / \mathrm{He}, \mathrm{O} 2 /(\mathrm{O} 2+\mathrm{He})=21 \%$ & 298 & 1 & 4.5 & 20 \\
\hline 59 & $\begin{array}{l}\text { 95/5\% CO/H2/air, } 15 \% \text { dil. with } \\
\mathrm{H} 20\end{array}$ & 323 & 1 & 0.6 & 10 & 138 & $\mathrm{H} 2 / \mathrm{O} 2 / \mathrm{He}, \mathrm{O} 2 /(\mathrm{O} 2+\mathrm{He})=0.125$ & 298 & 3 & 0.6 & 10 \\
\hline 60 & $\begin{array}{l}\text { 95/5\% CO/H2/air, 35\% dil. with } \\
\mathrm{H} 20\end{array}$ & 323 & 1 & 0.6 & 10 & 139 & $\mathrm{H} 2 / \mathrm{O} 2 / \mathrm{He}, \mathrm{O} 2 /(\mathrm{O} 2+\mathrm{He})=0.125$ & 298 & 3 & 1.5 & 10 \\
\hline 61 & $\begin{array}{l}\text { 95/5\% CO/H2/air, 0\% dil. with } \\
\mathrm{H} 20\end{array}$ & 323 & 1 & 0.9 & 10 & 140 & $\mathrm{H} 2 / \mathrm{O} 2 / \mathrm{He}, \mathrm{O} 2 /(\mathrm{O} 2+\mathrm{He})=0.125$ & 298 & 3 & 3.5 & 20 \\
\hline 62 & $\begin{array}{l}\text { 95/5\% CO/H2/air, } 15 \% \text { dil. with } \\
\mathrm{H} 20\end{array}$ & 323 & 1 & 0.9 & 10 & 141 & $\mathrm{H} 2 / \mathrm{O} 2 / \mathrm{He}, \mathrm{O} 2 /(\mathrm{O} 2+\mathrm{He})=0.125$ & 298 & 5 & 0.5 & 10 \\
\hline 63 & $\begin{array}{l}\text { 95/5\% CO/H2/air, 35\% dil. with } \\
\mathrm{H} 20\end{array}$ & 323 & 1 & 0.9 & 10 & 142 & $\mathrm{H} 2 / \mathrm{O} 2 / \mathrm{He}, \mathrm{O} 2 /(\mathrm{O} 2+\mathrm{He})=0.125$ & 298 & 5 & 1.5 & 10 \\
\hline 64 & $\begin{array}{l}\text { 80/20\% CO/H2/air, } 0 \% \text { dil. with } \\
\mathrm{H} 20\end{array}$ & 323 & 1 & 0.6 & 10 & 143 & $\mathrm{H} 2 / \mathrm{O} 2 / \mathrm{He}, \mathrm{O} 2 /(\mathrm{O} 2+\mathrm{He})=0.125$ & 298 & 5 & 3.5 & 20 \\
\hline 65 & $\begin{array}{l}80 / 20 \% \mathrm{CO} / \mathrm{H} 2 / \text { air, } 15 \% \text { dil. with } \\
\mathrm{H} 20\end{array}$ & 323 & 1 & 0.6 & 10 & 144 & $\mathrm{H} 2 / \mathrm{O} 2 / \mathrm{He}, \mathrm{O} 2 /(\mathrm{O} 2+\mathrm{He})=0.08$ & 298 & 10 & 0.75 & 10 \\
\hline 66 & $\begin{array}{l}\text { 80/20\% CO/H2/air, 35\% dil. with } \\
\mathrm{H} 20\end{array}$ & 323 & 1 & 0.6 & 10 & 145 & $\mathrm{H} 2 / \mathrm{O} 2 / \mathrm{He}, \mathrm{O} 2 /(\mathrm{O} 2+\mathrm{He})=0.08$ & 298 & 10 & 1.25 & 10 \\
\hline 67 & $50 \% \mathrm{H} 2-50 \% \mathrm{CO} / 90 \% \mathrm{He}-10 \% \mathrm{O} 2$ & 298 & 1 & 0.7 & 10 & 146 & $\mathrm{H} 2 / \mathrm{O} 2 / \mathrm{He}, \mathrm{O} 2 /(\mathrm{O} 2+\mathrm{He})=0.08$ & 298 & 10 & 2 & 10 \\
\hline 68 & $50 \% \mathrm{H} 2-50 \% \mathrm{CO} / 90 \% \mathrm{He}-10 \% \mathrm{O} 2$ & 298 & 5 & 0.7 & 10 & 147 & $\mathrm{H} 2 / \mathrm{O} 2 / \mathrm{He}, \mathrm{O} 2 /(\mathrm{O} 2+\mathrm{He})=0.08$ & 298 & 15 & 0.75 & 10 \\
\hline 69 & $50 \% \mathrm{H} 2-50 \% \mathrm{CO} / 90 \% \mathrm{~N} 2-10 \% \mathrm{O} 2$ & 298 & 1 & 1 & 10 & 148 & $\mathrm{H} 2 / \mathrm{O} 2 / \mathrm{He}, \mathrm{O} 2 /(\mathrm{O} 2+\mathrm{He})=0.08$ & 298 & 15 & 1.5 & 10 \\
\hline 70 & $50 \% \mathrm{H} 2-50 \% \mathrm{CO} / 90 \% \mathrm{~N} 2-10 \% \mathrm{O} 2$ & 298 & 3 & 1 & 10 & 149 & $\mathrm{H} 2 / \mathrm{O} 2 / \mathrm{He}, \mathrm{O} 2 /(\mathrm{O} 2+\mathrm{He})=0.08$ & 298 & 15 & 2 & 10 \\
\hline 71 & $\begin{array}{l}50 \% \mathrm{H} 2-50 \% \mathrm{CO} / 87.5 \% \mathrm{He}- \\
12.5 \% \mathrm{O} 2\end{array}$ & 298 & 1 & 0.6 & 10 & 150 & $\mathrm{H} 2 / \mathrm{O} 2 / \mathrm{He}, \mathrm{O} 2 /(\mathrm{O} 2+\mathrm{He})=0.08$ & 298 & 20 & 0.75 & 10 \\
\hline
\end{tabular}

14

American Institute of Aeronautics and Astronautics 


\begin{tabular}{|c|c|c|c|c|c|c|c|c|c|c|c|}
\hline \# & Mixture & $\mathrm{T}_{0}, \mathrm{~K}$ & $\begin{array}{l}\mathrm{P}, \\
\text { bar }\end{array}$ & $\varphi$ & $\begin{array}{l}\text { Estim } \\
\text { ated } \\
\text { error, } \\
\%\end{array}$ & $\#$ & Mixture & $\mathrm{T}_{0}, \mathrm{~K}$ & $\begin{array}{l}\mathrm{P}, \\
\text { bar }\end{array}$ & $\varphi$ & $\begin{array}{l}\text { Estim } \\
\text { ated } \\
\text { error, } \\
\%\end{array}$ \\
\hline 72 & $\begin{array}{l}50 \% \mathrm{H} 2-50 \% \mathrm{CO} / 87.5 \% \mathrm{He}- \\
12.5 \% \mathrm{O} 2\end{array}$ & 298 & 3 & 0.6 & 10 & 151 & $\mathrm{H} 2 / \mathrm{O} 2 / \mathrm{He}, \mathrm{O} 2 /(\mathrm{O} 2+\mathrm{He})=0.08$ & 298 & 20 & 1.5 & 10 \\
\hline 73 & $\begin{array}{l}50 \% \mathrm{H} 2-50 \% \mathrm{CO} / 87.5 \% \mathrm{He}- \\
12.5 \% \mathrm{O} 2\end{array}$ & 298 & 9 & 0.6 & 15 & 152 & $\mathrm{H} 2 / \mathrm{O} 2 / \mathrm{He}, \mathrm{O} 2 /(\mathrm{O} 2+\mathrm{He})=0.08$ & 298 & 20 & 2 & 10 \\
\hline 74 & $\begin{array}{l}85 \% \mathrm{H} 2-15 \% \mathrm{CO} / 12.5 \% \mathrm{O} 2- \\
87.5 \% \mathrm{He}\end{array}$ & 298 & 1 & 0.5 & 10 & 153 & $\mathrm{H} 2 / \mathrm{O} 2,50 \% \mathrm{CO} 2$ dil. & 298 & 1 & 0.6 & 10 \\
\hline 75 & $\begin{array}{l}85 \% \mathrm{H} 2-15 \% \mathrm{CO} / 12.5 \% \mathrm{O} 2- \\
87.5 \% \mathrm{He}\end{array}$ & 298 & 5 & 0.5 & 10 & 154 & $\mathrm{H} 2 / \mathrm{O} 2,50 \% \mathrm{CO} 2$ dil. & 298 & 1 & 1.2 & 10 \\
\hline 76 & $\begin{array}{l}85 \% \mathrm{H} 2-15 \% \mathrm{CO} / 12.5 \% \mathrm{O} 2- \\
87.5 \% \mathrm{He}\end{array}$ & 298 & 10 & 0.5 & 15 & 155 & $\mathrm{H} 2 / \mathrm{O} 2,50 \% \mathrm{CO} 2$ dil. & 298 & 1 & 2 & 10 \\
\hline 77 & $\begin{array}{l}85 \% \mathrm{H} 2-15 \% \mathrm{CO} / 12.5 \% \mathrm{O} 2- \\
87.5 \% \mathrm{He}\end{array}$ & 298 & 1 & 0.5 & 10 & 156 & $\mathrm{H} 2 / \mathrm{O} 2,50 \% \mathrm{~N} 2$ dil. & 298 & 1 & 0.6 & 10 \\
\hline 78 & $\begin{array}{l}85 \% \mathrm{H} 2-15 \% \mathrm{CO} / 12.5 \% \mathrm{O} 2- \\
87.5 \% \mathrm{He}\end{array}$ & 298 & 4 & 0.5 & 10 & 157 & $\mathrm{H} 2 / \mathrm{O} 2,50 \% \mathrm{~N} 2$ dil. & 298 & 1 & 1.2 & 10 \\
\hline 79 & $\begin{array}{l}85 \% \mathrm{H} 2-15 \% \mathrm{CO} / 12.5 \% \mathrm{O} 2- \\
87.5 \% \mathrm{He}\end{array}$ & 298 & 10 & 0.5 & 15 & 158 & $\mathrm{H} 2 / \mathrm{O} 2,50 \% \mathrm{~N} 2$ dil. & 298 & 1 & 2 & 10 \\
\hline
\end{tabular}

Appendix 3. List of QoIs suggested by VCM

Ignition delay QoIs

\begin{tabular}{|c|c|c|c|c|c|c|c|c|c|c|c|}
\hline \# & Target ID & $\begin{array}{c}\text { LB } \\
\text { change, } \\
\text { ms }\end{array}$ & $\begin{array}{c}\text { UB } \\
\text { change, } \\
\text { ms }\end{array}$ & \# & Target ID & $\begin{array}{c}\text { LB } \\
\text { change, } \\
\text { ms }\end{array}$ & $\begin{array}{c}\text { UB } \\
\text { change, } \\
\text { ms }\end{array}$ & \# & Target ID & $\begin{array}{c}\text { LB } \\
\text { change, } \\
\text { ms }\end{array}$ & $\begin{array}{c}\text { UB } \\
\text { change, } \\
\text { ms }\end{array}$ \\
\hline 1 & $\mathrm{a} 00000110$ & -0.4774 & 0.0000 & 29 & $\mathrm{a} 00000498$ & $\begin{array}{l}-0.0017 \\
\end{array}$ & 0.0000 & 57 & $\mathrm{a} 00000540$ & 0.0000 & 0.1910 \\
\hline 2 & $\mathrm{a} 00000194$ & -0.1406 & 0.0000 & 30 & $\mathrm{a} 00000491$ & -0.6858 & 0.0000 & 58 & $\mathrm{a} 00000552$ & 0.0000 & 0.0261 \\
\hline 3 & $\mathrm{a} 00000228$ & -0.2355 & 0.0000 & 31 & $\mathrm{a} 00000492$ & -0.0097 & 0.0000 & 59 & $\mathrm{a} 00000555$ & 0.0000 & 0.5183 \\
\hline 4 & $\mathrm{a} 00000235$ & -0.5238 & 0.0000 & 32 & a00000495 & -0.5137 & 0.0000 & 60 & $\mathrm{a} 00000570$ & 0.0000 & 0.0719 \\
\hline 5 & $\mathrm{a} 00000236$ & -0.3754 & 0.0000 & 33 & $\mathrm{a} 00000496$ & -0.3855 & 0.0000 & 61 & $\mathrm{a} 00000580$ & 0.0000 & 1.1304 \\
\hline 6 & $\mathrm{a} 00000237$ & -0.2418 & 0.0000 & 34 & a00000497 & -0.0217 & 0.0000 & 62 & $\mathrm{a} 00000591$ & 0.0000 & 0.0795 \\
\hline 7 & $\mathrm{a} 00000240$ & -0.0606 & 0.0000 & 35 & $\mathrm{a} 00000150$ & 0.0000 & 0.0709 & 63 & $\mathrm{a} 00000592$ & 0.0000 & 0.1835 \\
\hline 8 & $\mathrm{a} 00000243$ & -0.0077 & 0.0000 & 36 & $\mathrm{a} 00000152$ & 0.0000 & 0.0262 & 64 & $\mathrm{a} 00000593$ & 0.0000 & 0.2893 \\
\hline 9 & a00000318 & -0.1501 & 0.0000 & 37 & $\mathrm{a} 00000153$ & 0.0000 & 0.0026 & 65 & $\mathrm{a} 00000595$ & 0.0000 & 1.1639 \\
\hline 10 & $\mathrm{a} 00000547$ & -0.1812 & 0.0000 & 38 & $\mathrm{a} 00000154$ & 0.0000 & 0.0417 & 66 & $\mathrm{a} 00000600$ & 0.0000 & 2.4465 \\
\hline 11 & $\mathrm{a} 00000548$ & -0.0016 & 0.0000 & 39 & $\mathrm{a} 00000155$ & 0.0000 & 0.0472 & 67 & $\mathrm{a} 00000612$ & 0.0000 & 6.4691 \\
\hline 12 & a00000549 & -0.1356 & 0.0000 & 40 & $\mathrm{a} 00000107$ & 0.0000 & 0.0052 & 68 & a00000619 & 0.0000 & 3.8471 \\
\hline 13 & $\mathrm{a} 00000558$ & -0.0035 & 0.0000 & 41 & a00000136 & 0.0000 & 0.0051 & 69 & $\mathrm{a} 00000620$ & 0.0000 & 0.0071 \\
\hline 14 & $\mathrm{a} 00000582$ & -0.0360 & 0.0000 & 42 & a00000179 & 0.0000 & 0.8560 & 70 & $\mathrm{a} 00000631$ & 0.0000 & 2.8933 \\
\hline 15 & $\mathrm{a} 00000623$ & -0.3518 & 0.0000 & 43 & a00000186 & 0.0000 & 0.0097 & 71 & a00000644 & 0.0000 & 0.0068 \\
\hline 16 & a00000624 & -0.5036 & 0.0000 & 44 & $\mathrm{a} 00000191$ & 0.0000 & 9.1044 & 72 & $\mathrm{a} 00000645$ & 0.0000 & 0.0029 \\
\hline 17 & $\mathrm{a} 00000633$ & -0.0073 & 0.0000 & 45 & $\mathrm{a} 00000196$ & 0.0000 & 0.0043 & 73 & $\mathrm{a} 00000653$ & 0.0000 & 0.0002 \\
\hline 18 & a00000635 & -0.1842 & 0.0000 & 46 & a00000310 & 0.0000 & 0.9045 & 74 & a00000673 & 0.0000 & 0.0336 \\
\hline 19 & a00000647 & -0.4052 & 0.0000 & 47 & $\mathrm{a} 00000311$ & 0.0000 & 0.0128 & 75 & a00000686 & 0.0000 & 13.2658 \\
\hline 20 & a00000649 & -0.0042 & 0.0000 & 48 & $\mathrm{a} 00000312$ & 0.0000 & 0.0136 & 76 & $\mathrm{a} 00000692$ & 0.0000 & 0.5164 \\
\hline 21 & a00000650 & -0.1770 & 0.0000 & 49 & $\mathrm{a} 00000316$ & 0.0000 & 2.8089 & 77 & $\mathrm{a} 00000698$ & 0.0000 & 0.0014 \\
\hline 22 & $\mathrm{a} 00000662$ & -0.0034 & 0.0000 & 50 & $\mathrm{a} 00000322$ & 0.0000 & 1.6876 & 78 & a00000699 & 0.0000 & 0.0000 \\
\hline 23 & a00000663 & -0.1294 & 0.0000 & 51 & $\mathrm{a} 00000335$ & 0.0000 & 0.0052 & 79 & $\mathrm{a} 00000700$ & 0.0000 & 1.3386 \\
\hline 24 & $\mathrm{a} 00000664$ & -0.0087 & 0.0000 & 52 & $\mathrm{a} 00000353$ & 0.0000 & 0.0406 & 80 & $\mathrm{a} 00000704$ & 0.0000 & 0.0061 \\
\hline 25 & $\mathrm{a} 00000665$ & -0.0024 & 0.0000 & 53 & $\mathrm{a} 00000354$ & 0.0000 & 0.8257 & 81 & $\mathrm{a} 00000710$ & 0.0000 & 0.0119 \\
\hline 26 & a00000677 & -0.1225 & 0.0000 & 54 & $\mathrm{a} 00000356$ & 0.0000 & 0.0408 & 82 & a00000608 & 0.0000 & 0.0007 \\
\hline 27 & $\mathrm{a} 00000682$ & -0.0011 & 0.0000 & 55 & $\mathrm{a} 00000357$ & 0.0000 & 0.5274 & 83 & $\mathrm{a} 00000713$ & 0.0000 & 0.0001 \\
\hline 28 & $\mathrm{a} 00000703$ & -0.0800 & 0.0000 & 56 & $\mathrm{a} 00000538$ & 0.0000 & 0.0150 & 84 & $\mathrm{a} 00000505$ & 0.0000 & 0.0118 \\
\hline \multicolumn{12}{|c|}{ Laminar flame speed QoIs } \\
\hline \# & Flame ID & $\begin{array}{c}\text { LB } \\
\text { change, } \\
\mathrm{cm} / \mathrm{s}\end{array}$ & $\begin{array}{c}\text { UB } \\
\text { change, } \\
\mathrm{cm} / \mathrm{s}\end{array}$ & $\#$ & Flame ID & $\begin{array}{c}\mathrm{LB} \\
\text { change, } \\
\mathrm{cm} / \mathrm{s}\end{array}$ & $\begin{array}{c}\text { UB } \\
\text { change, } \\
\mathrm{cm} / \mathrm{s}\end{array}$ & \# & Flame ID & $\begin{array}{c}\mathrm{LB} \\
\text { change, } \\
\mathrm{cm} / \mathrm{s}\end{array}$ & $\begin{array}{c}\text { UB } \\
\text { change, } \\
\mathrm{cm} / \mathrm{s}\end{array}$ \\
\hline 1 & $\begin{array}{c}\text { Flame QoI } \\
67\end{array}$ & 0.0000 & 0.1786 & 2 & $\begin{array}{c}\text { Flame QoI } \\
68\end{array}$ & -3.8602 & 0.0000 & 3 & $\begin{array}{c}\text { Flame QoI } \\
112\end{array}$ & 0.0000 & 1.7301 \\
\hline
\end{tabular}

\section{Acknowledgments}

The authors from the University of California, Berkeley, acknowledge the support of the National Nuclear Security Administration, U.S. Department of Energy, under Award DE-NA0002375. The views and opinions of authors expressed herein do not necessarily state or reflect those of the United States Government or any agency thereof. 


\section{References}

${ }^{1}$ Frenklach, M. "Transforming data into knowledge-Process Informatics for combustion chemistry," Proc. Combust. Inst. Vol. 31, No. 1, 2007, pp. 125-140.

${ }^{2}$ Frenklach, M., Packard, A., and Feeley, R. "Optimization of reaction models with Solution Mapping," Modeling of Chemical Reactions. Elsevier, New York, 2007, pp. 243-291.

${ }^{3}$ Smith, G. P., Golden, D. M., Frenklach, M., Moriarty, N. W., Eiteneer, B., Goldenberg, M., Bowman, T., Hanson, R. K., Song, S., Gardiner, W. C., Jr., Lissianski, V. V., and Qin, Z. "GRI-Mech 3.0," http://www.me.berkeley.edu/gri_mech/.

${ }^{4}$ Kéromnès, A., Metcalfe, W. K., Heufer, K. A., Donohoe, N., Das, A. K., Sung, C.-J., Herzler, J., Naumann, C., Griebel, P., Mathieu, O., Krejci, M. C., Petersen, E. L., Pitz, W. J., and Curran, H. J. "An experimental and detailed chemical kinetic modeling study of hydrogen and syngas mixture oxidation at elevated pressures," Combust. Flame Vol. 160, No. 6, 2013 , pp. 995-1011.

${ }^{5}$ Turányi, T., Nagy, T., Zsély, I. G., Cserháti, M., Varga, T., Szabó, B. T., Sedyó, I., Kiss, P. T., Zempléni, A., and Curran, H. J. "Determination of rate parameters based on both direct and indirect measurements," Int. J. Chem. Kinet. Vol. 44, No. 5, 2012, pp. 284-302.

${ }^{6}$ Varga, T., Olm, C., Nagy, T., Zsély, I. G., Valkó, É., Pálvölgyi, R., Curran, H. J., and Turányi, T. "Development of a joint hydrogen and syngas combustion mechanism based on an optimization approach," Int. J. Chem. Kinet. Vol. 48, No. 8, 2016, pp. 407-422.

${ }^{7}$ Frenklach, M. "PrIMe," https://prime.cki-know.org/.

${ }^{8}$ Frenklach, M., Packard, A., Seiler, P., and Feeley, R. "Collaborative data processing in developing predictive models of complex reaction systems," Int. J. Chem. Kinet. Vol. 36, No. 1, 2004, pp. 57-66.

${ }^{9}$ You, X., Packard, A., and Frenklach, M. "Process informatics tools for predictive modeling: Hydrogen combustion," Int. J. Chem. Kinet. Vol. 44, No. 2, 2012, pp. 101-116.

${ }^{10}$ Slavinskaya, N. A., Abbasi, M., Starcke, J. H., Whitside, R., Mirzayeva, A., Riedel, U., Li, W., Oreluk, J., Hegde, A., Packard, A., Frenklach, M., Gerasimov, G., and Shatalov, O. "Development of an Uncertainty Quantification Predictive Chemical Reaction Model for Syngas Combustion," Energy \& Fuels Vol. 31, No. 3, 2017, pp. 2274-2297.

${ }^{11}$ Feeley, R., Seiler, P., Packard, A., and Frenklach, M. "Consistency of a reaction dataset," J. Phys. Chem. A Vol. 108, No. 44, 2004, pp. 9573-9583.

${ }^{12}$ Russi, T., Packard, A., Feeley, R., and Frenklach, M. "Sensitivity analysis of uncertainty in model prediction," J. Phys. Chem. A Vol. 112, No. 12, 2008, pp. 2579-2588.

${ }^{13}$ Russi, T., Packard, A., and Frenklach, M. "Uncertainty quantification: Making predictions of complex reaction systems reliable," Chem. Phys. Lett. Vol. 499, No. 1-3, 2010, pp. 1-8.

${ }^{14}$ Seiler, P., Frenklach, M., Packard, A., and Feeley, R. "Numerical approaches for collaborative data processing," Optim. Eng. Vol. 7, No. 4, 2006, pp. 459-478.

${ }^{15}$ Frenklach, M., Packard, A., and Seiler, P. "Prediction uncertainty from models and data," IEEE. Anchorage, Alaska, 2002, pp. 4135-4140.

${ }^{16}$ Hegde, A., Li, W., Oreluk, J., Packard, A., and Frenklach, M. "Consistency analysis for massively inconsistent datasets in Bound-to-Bound Data Collaboration." preparation.

${ }^{17}$ You, X., Russi, T., Packard, A., and Frenklach, M. "Optimization of combustion kinetic models on a feasible set," Proc. Combust. Inst. Vol. 33, No. 1, 2011, pp. 509-516.

${ }^{18}$ Wang, H., and Sheen, D. A. "Combustion kinetic model uncertainty quantification, propagation and minimization," Prog. Energy Combust. Sci. Vol. 47, 2015, pp. 1-31.

${ }^{19}$ Frenklach, M. "Modeling," Combustion Chemistry. Springer-Verlag, New York, 1984, pp. 423-453.

${ }^{20}$ Kalitan, D. M., Mertens, J. D., Crofton, M. W., and Petersen, E. L. "Ignition and oxidation of lean CO/H $\mathrm{H}_{2}$ fuel blends in air," J. Propul. Power Vol. 23, No. 6, 2007, pp. 1291-1301.

${ }^{21}$ Mathieu, O., Kopp, M. M., and Petersen, E. L. "Shock-tube study of the ignition of multi-component syngas mixtures with and without ammonia impurities," Proc. Combust. Inst. Vol. 34, No. 2, 2013, pp. 3211-3218.

${ }^{22}$ Herzler, J., and Naumann, C. "Shock-tube study of the ignition of methane/ethane/hydrogen mixtures with hydrogen contents from $0 \%$ to $100 \%$ at different pressures," Proceedings of the Combustion Institute Vol. 32, No. 1, 2009 , pp. $213-220$.

${ }^{23}$ Petersen, E. L., Kalitan, D. M., Barrett, A. B., Reehal, S. C., Mertens, J. D., Beerer, D. J., Hack, R. L., and McDonell, V. G. "New syngas/air ignition data at lower temperature and elevated pressure and comparison to current kinetics models," Combust. Flame Vol. 149, No. 1-2, 2007, pp. 244-247.

${ }^{24}$ Vasu, S. S., Davidson, D. F., and Hanson, R. K. "Shock tube study of syngas ignition in rich $\mathrm{CO}_{2}$ mixtures and determination of the rate of $\mathrm{H}+\mathrm{O}_{2}+\mathrm{CO}_{2} \rightarrow \mathrm{HO}_{2}+\mathrm{CO}_{2}$," Energy Fuels Vol. 25, No. 3, 2011, pp. 990-997.

${ }^{25}$ Naumann, C., Herzler, J., Griebel, P., Curran, H. J., Keromnes, A., and Mantzaras, I. "Low Emission Gas Turbine Technology for Hydrogen-rich Syngas. H2-IGCC project - Deliverable 1.1.3 report.," 7th Framework Programme FP7ENERGY-2008-TREN-1, ENERGY-2008-6-CLEAN COAL TECHNOLOGIES. 2011.

${ }^{26}$ Mertens, J. D., Kalitan, D. M., Barrett, A. B., and Petersen, E. L. "Determination of the rate of $\mathrm{H}_{+} \mathrm{O}_{2}+\mathrm{M} \rightarrow \mathrm{HO}_{2}+\mathrm{M}\left(\mathrm{M}=\mathrm{N}_{2}\right.$, $\mathrm{Ar}, \mathrm{H}_{2} \mathrm{O}$ ) from ignition of syngas at practical conditions," Proc. Combust. Inst. Vol. 32, No. 1, 2009, pp. $295-303$.

${ }^{27}$ Thi, L. D., Zhang, Y., and Huang, Z. "Shock tube study on ignition delay of multi-component syngas mixtures - Effect of equivalence ratio," Int. J. Hydrogen Energy Vol. 39, No. 11, 2014, pp. 6034-6043.

${ }^{28}$ Pan, L., Zhang, Y., Zhang, J., Tian, Z., and Huang, Z. "Shock tube and kinetic study of C2H6/H2/O2/Ar mixtures at elevated pressures," International Journal of Hydrogen Energy Vol. 39, No. 11, 2014, pp. 6024-6033.

16

American Institute of Aeronautics and Astronautics 
${ }^{29}$ Mittal, G., Sung, C.-J., and Yetter, R. A. "Autoignition of $\mathrm{H}_{2} / \mathrm{CO}$ at elevated pressures in a rapid compression machine," Int. J. Chem. Kinet. Vol. 38, No. 8, 2006, pp. 516-529.

${ }^{30}$ Schultz, E. S., J. "Validation of Detailed Reaction Mechanisms for Detonation Simulation," Explosion Dynamics Laboratory Report FM99-5. California Institute of Technology, Pasadena, CA, 2000.

${ }^{31}$ Petersen, E. L. D., D.F.; Rohrig, M.; Hanson, R.K. "High pressure shock tube measurements of ignition times in stoichiometric $\mathrm{H}_{2} / \mathrm{O}_{2} /$ Ar mixtures," 20th International symposium on shock waves. 1996, pp. 941-6.

${ }^{32}$ Zhang, Y., Huang, Z., Wei, L., Zhang, J., and Law, C. K. "Experimental and modeling study on ignition delays of lean mixtures of methane, hydrogen, oxygen, and argon at elevated pressures," Combustion and Flame Vol. 159, No. 3, 2012, pp. 918-931.

${ }^{33}$ Herzler, J., and Naumann, C. "Shock tube study of the ignition of lean $\mathrm{CO} / \mathrm{H}_{2}$ fuel blends at intermediate temperatures and high pressure," Combust. Sci. Technol. Vol. 180, No. 10-11, 2008, pp. 2015-2028.

${ }^{34}$ Pang, G. A., Davidson, D. F., and Hanson, R. K. "Experimental study and modeling of shock tube ignition delay times for hydrogen-oxygen-argon mixtures at low temperatures," Proceedings of the Combustion Institute Vol. 32, No. 1, 2009, pp. 181188.

${ }^{35}$ Slack, M. W. "Rate Coefficient for $\mathrm{H}+\mathrm{O}_{2}+\mathrm{M}=\mathrm{HO}_{2}+\mathrm{M}$ Evaluated from Shock Tube Measurements of Induction Times," Combustion and Flame Vol. 28, 1977, pp. 241-249.

${ }^{36}$ Krejci, M. C., Mathieu, O., Vissotski, A. J., Ravi, S., Sikes, T. G., Petersen, E. L., Kérmonès, A., Metcalfe, W., and Curran, H. J. "Laminar flame speed and ignition delay time data for the kinetic modeling of hydrogen and syngas fuel blends," J. Eng. Gas Turbines Power Vol. 135, No. 2, 2013, p. 021503.

${ }^{37}$ Sun, H., Yang, S. I., Jomaas, G., and Law, C. K. "High-pressure laminar flame speeds and kinetic modeling of carbon monoxide/hydrogen combustion," Proc. Combust. Inst. Vol. 31, No. 1, 2007, pp. 439-446.

${ }^{38}$ Goswami, M., van Griensven, J. G. H., Bastiaans, R. J. M., Konnov, A. A., and Goey, L. P. H. "Experimental and modeling study of the effect of elevated pressure on lean high-hydrogen syngas flames," Proc. Combust. Inst. Vol. 35, No. 1, 2015, pp. 655-662.

${ }^{39}$ Wang, Z. H., Weng, W. B., He, Y., Li, Z. S., and Cen, K. F. "Effect of $\mathrm{H}_{2} / \mathrm{CO}$ ratio and $\mathrm{N}_{2} / \mathrm{CO}_{2}$ dilution rate on laminar burning velocity of syngas investigated by direct measurement and simulation," Fuel Vol. 141, 2015, pp. 285-292.

${ }^{40}$ Hassan, M. I., Aung, K. T., and Faeth, G. M. "Properties of Laminar Premixed CO/H/Air Flames at Various Pressures," $J$. Propul. Power Vol. 13, No. 2, 1997, pp. 239-245.

${ }^{41}$ Zhang, W., Gou, X., Kong, W., and Chen, Z. "Laminar flame speeds of lean high-hydrogen syngas at normal and elevated pressures," Fuel Vol. 181, 2016, pp. 958-963.

${ }^{42}$ Weng, W. B., Wang, Z. H., He, Y., Whiddon, R., Zhou, Y. J., Li, Z. S., and Cen, K. F. "Effect of $\mathrm{N}_{2} / \mathrm{CO}_{2}$ dilution on laminar burning velocity of $\mathrm{H}_{2}-\mathrm{CO}-\mathrm{O}_{2}$ oxy-fuel premixed flame," Int. J. Hydrogen Energy Vol. 40, No. 2, 2015, pp. 1203-1211.

${ }^{43}$ Natarajan, J., Lieuwen, T., and Seitzman, J. "Laminar flame speeds of $\mathrm{H}_{2} / \mathrm{CO}$ mixtures: Effect of $\mathrm{CO}_{2}$ dilution, preheat temperature, and pressure," Combust. Flame Vol. 151, No. 1-2, 2007, pp. 104-119.

${ }^{44}$ Han, M., Ai, Y., Chen, Z., and Kong, W. "Laminar flame speeds of $\mathrm{H}_{2} / \mathrm{CO}$ with $\mathrm{CO}_{2}$ dilution at normal and elevated pressures and temperatures," Fuel Vol. 148, 2015, pp. 32-38.

${ }^{45}$ Xie, Y., Wang, J., Xu, N., Yu, S., Zhang, M., and Huang, Z. "Thermal and chemical effects of water addition on laminar burning velocity of syngas," Energy Fuels Vol. 28, No. 5, 2014, pp. 3391-3398.

${ }^{46}$ Natarajan, J., Kochar, Y., Lieuwen, T., and Seitzman, J. "Pressure and preheat dependence of laminar flame speeds of $\mathrm{H}_{2} / \mathrm{CO} / \mathrm{CO}_{2} / \mathrm{O}_{2} / \mathrm{He}$ mixtures," Proc. Combust. Inst. Vol. 32, No. 1, 2009, pp. 1261-1268.

${ }^{47}$ Lapalme, D., and Seers, P. "Influence of $\mathrm{CO}_{2}, \mathrm{CH}_{4}$, and initial temperature on $\mathrm{H}_{2} / \mathrm{CO}$ laminar flame speed," Int. J. Hydrogen Energy Vol. 39, No. 7, 2014, pp. 3477-3486.

${ }^{48}$ Xie, Y., Wang, J., Xu, N., Yu, S., and Huang, Z. "Comparative study on the effect of $\mathrm{CO}_{2}$ and $\mathrm{H}_{2} \mathrm{O}$ dilution on laminar burning characteristics of CO/ $\mathrm{H}_{2}$ /air mixtures," Int. J. Hydrogen Energy Vol. 39, No. 7, 2014, pp. 3450-3458.

${ }^{49}$ Das, A. K., Kumar, K., and Sung, C.-J. "Laminar flame speeds of moist syngas mixtures," Combust. Flame Vol. 158, No. 2, 2011, pp. 345-353.

${ }^{50}$ Li, H.-M., Li, G.-X., Sun, Z.-Y., Zhou, Z.-H., Li, Y., and Yuan, Y. "Effect of dilution on laminar burning characteristics of $\mathrm{H}_{2} / \mathrm{CO} / \mathrm{CO}_{2} /$ air premixed flames with various hydrogen fractions," Exp. Therm. Fluid Sci. Vol. 74, 2016, pp. 160-168.

${ }^{51}$ Aung, K. T., Hassan, M. I., and Faeth, G. M. "Flame Stretch Interactions of Laminar Premixed Hydrogen/Air Flames at Normal Temperature and Pressure," Combust. Flame Vol. 109, 1997, pp. 1-24.

${ }^{52}$ Taylor, S. C. "Burning Velocity and the Influence of Flame Stretch." Ph.D. thesis, University of Leeds, 1991.

${ }^{53}$ Egolfopoulos, F. N., and Law, C. K. "An experimental and computational study of the burning rates of ultra-lean to moderately-rich H2/O2/N2 laminar flames with pressure variations," Proc. Combust. Inst. Vol. 23, 1990, p. 333.

${ }^{54}$ Law, C. K. "A Compilation of Experimental Data on Laminar Burning Velocities," Reduced Kinetic Mechanisms for Applications in Combustion Systems Springer-Verlag, Berlin, 1993, p. 15.

${ }^{55}$ Vagelopoulos, C. M., Egolfopoulos, F. N., and Law, C. K. "Further considerations on the determination of laminar flame speeds with the counterflow twin-flame technique," Proc. Combust. Inst. Vol. 25, No. 1, 1994, pp. 1341-1347.

${ }^{56} \mathrm{Hu}$, E., Huang, Z., He, J., Jin, C., and Zheng, J. "Experimental and numerical study on laminar burning characteristics of premixed methane-hydrogen-air flames," International Journal of Hydrogen Energy Vol. 34, No. 11, 2009, pp. 4876-4888.

${ }^{57}$ Tang, C., Huang, Z., Jin, C., He, J., Wang, J., Wang, X., and Miao, H. "Laminar burning velocities and combustion characteristics of propane-hydrogen-air premixed flames," International Journal of Hydrogen Energy Vol. 33, No. 18, 2008, pp. 4906-4914. 
${ }^{58}$ Verhelst, S., Woolley, R., Lawes, M., and Sierens, R. "Laminar and unstable burning velocities and Markstein lengths of hydrogen-air mixtures at engine-like conditions," Proceedings of the Combustion Institute Vol. 30, No. 1, 2005 , pp. $209-216$.

${ }^{59}$ Kwon, O. C., and Faeth, G. M. "Flame/stretch interactions of premixed hydrogen-fueled flames: Measurements and predictions," Combust. Flame Vol. 124, No. 4, 2001, pp. 590-610.

${ }^{60}$ Tse, S. D., Zhu, D. L., and Law, C. K. "Morphology and burning rates of expanding spherical flames in $\mathrm{H}_{2} / \mathrm{O}_{2} / \mathrm{inert}$ mixtures up to 60 atmospheres," Proc. Combust. Inst. Vol. 28, 2000, p. 1793.

${ }^{61}$ Goswami, M., Bastiaans, R. J. M., Konnov, A. A., and Goey, L. P. H. "Laminar burning velocity of lean $\mathrm{H}_{2}-\mathrm{CO}$ mixtures at elevated pressure using the heat flux method," Int. J. Hydrogen Energy Vol. 39, No. 3, 2014, pp. 1485-1498.

${ }^{62}$ Sun, S., Meng, S., Zhao, Y., Xu, H., Guo, Y., and Qin, Y. "Experimental and theoretical studies of laminar flame speed of $\mathrm{CO} / \mathrm{H}_{2}$ in $\mathrm{O}_{2} / \mathrm{H}_{2} \mathrm{O}$ atmosphere," Int. J. Hydrogen Energy Vol. 41, No. 4, 2016, pp. 3272-3283. 